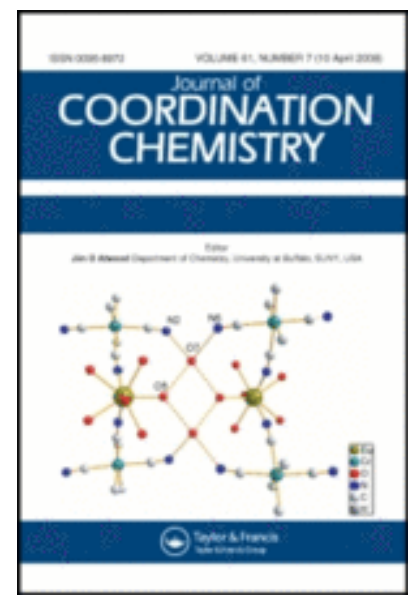

\title{
Synthesis and structure of organoplatinum(II) complexes containing aryl olefins and 8-hydroxyquinolines
}

\begin{tabular}{|c|c|}
\hline Journal: & Journal of Coordination Chemistry \\
\hline Manuscript ID & GCOO-2018-0484.R1 \\
\hline Manuscript Type: & Original Paper \\
\hline $\begin{array}{l}\text { Date Submitted by the } \\
\text { Author: }\end{array}$ & $\mathrm{n} / \mathrm{a}$ \\
\hline Complete List of Authors: & $\begin{array}{l}\text { Hong Hai, Le; Hanoi National University of Education, Chemistry } \\
\text { Ngoc Vinh, Nguyen; Hanoi National University of Education } \\
\text { tuyen, luu; Hanoi National University of Education } \\
\text { meervelt, luc; KU Leuven Belgium } \\
\text { Da, Tran; Hanoi National University of Education, Chemistry }\end{array}$ \\
\hline Keywords: & $\begin{array}{l}\text { Platinum(II) complex, Organoplatinum, Safrole, Eugenol, 8- } \\
\text { Hydroxyquinolines }\end{array}$ \\
\hline \multicolumn{2}{|c|}{$\begin{array}{l}\text { Note: The following files were submitted by the author for peer review, but cannot be converted to PDF } \\
\text { You must view these files (e.g. movies) online. }\end{array}$} \\
\hline HAI39. cif & \\
\hline
\end{tabular}

\section{SCHOLARONE $^{\text {m }}$ Manuscripts}


Synthesis and structure of organoplatinum(II) complexes containing aryl olefins and 8-hydroxyquinolines

Le Thi Hong Hai, Nguyen Thi Ngoc Vinh, Tran Thi Da, Luc Van Meervelt

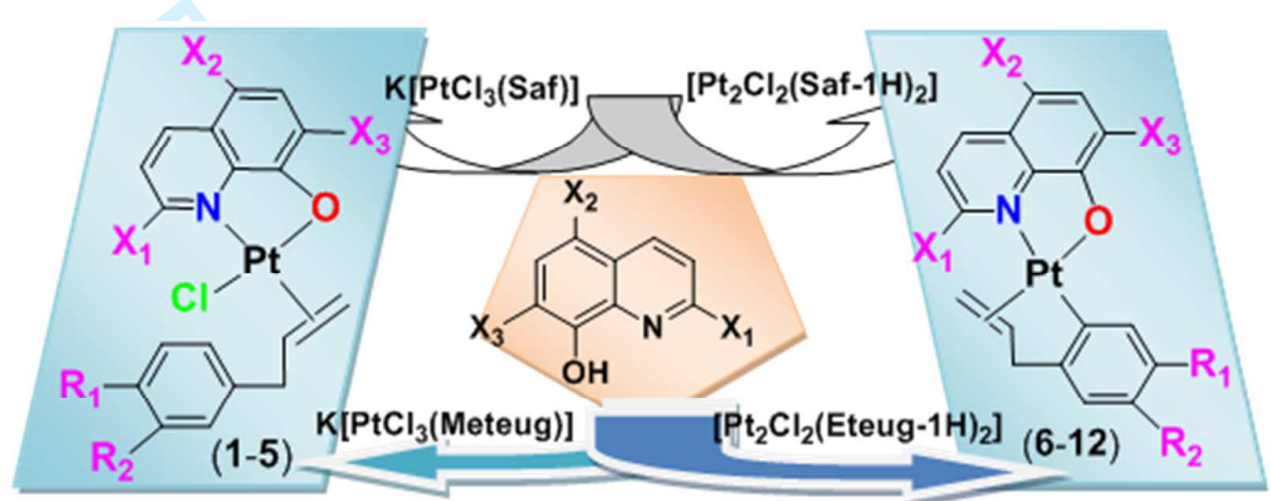




\title{
Synthesis and structure of organoplatinum(II) complexes containing aryl olefins and 8-hydroxyquinolines
}

Le Thi Hong Hai ${ }^{1}$, Nguyen Thi Ngoc Vinh ${ }^{1}$, Luu Thi Tuyen ${ }^{1}$, Luc Van Meervelt ${ }^{2}$, Tran Thị Da ${ }^{1}$ ${ }^{1}$ Department of Chemistry, Hanoi National University of Education, 136 Xuan Thuy, Hanoi, Vietnam.

${ }^{2}$ Biomolecular Architecture, Chemistry Department KU Leuven, Celestijnenlaan $200 F$ box 2404, B-3001 Leuven, Belgium.

\begin{abstract}
Twelve new organoplatinum(II) complexes: [PtCl(Saf)(2-Me-8-OquiN)] [PtCl(Saf)(5,7-Cl $-8-O q u i N)](2),\left[\mathrm{PtCl}(\mathrm{Saf})\left(5,7-\mathrm{Cl}_{2}-2-\mathrm{Me}-8-\mathrm{OquiN}\right)\right](3),[\mathrm{PtCl}(\mathrm{Meteug})(5,7-$ $\mathrm{Cl}_{2}$-8-OquiN)] (4), [PtCl(Meteug)(5,7-Cl $-2-\mathrm{Me}-8$-OquiN)] (5), [Pt(Saf-1H)(2-Me-8-OquiN)] (6), $\left[\mathrm{Pt}(\mathrm{Saf}-1 \mathrm{H})\left(5,7-\mathrm{Cl}_{2}-8-\mathrm{OquiN}\right)\right](7),\left[\mathrm{Pt}(\mathrm{Saf}-1 \mathrm{H})\left(5,7-\mathrm{Cl}_{2}-2-\mathrm{Me}-8-\mathrm{OquiN}\right)\right](\mathbf{8})$, Pt(Eteug1H)(2-Me-8-OquiN)] (9),[Pt(Eteug-1H)(2-OHC-8-OquiN)] (10), $[\mathrm{Pt}($ Eteug-1H)(5,7-Cl $2-8$ OquiN)] (11), and [Pt(Eteug-1H)(5,7-Cl $2-2-M e-8-O q u i N)]$ (12) (Saf: Safrole, Meteug: Methyl eugenoxyacetate, Saf-1H: Deprotonated safrole, Eteug-1H: Deprotonated ethyl eugenoxyacetate, OquiN: substituted quinolin-8-olate) were synthesized. These complexes were characterized by elemental analyses, IR, ${ }^{1} \mathrm{H}$ NMR, for several also by NOESY, MS spectra and single crystal X-ray diffraction. It is shown that in $\mathbf{1 - 5}$ the $\mathrm{Pt}(\mathrm{II})$ atom is surrounded by a $\mathrm{Cl}$ atom and the ethylenic double bond of the aryl olefin, and by $\mathrm{N}$ and $\mathrm{O}$ atoms of the 8-OquiN, its donor $\mathrm{N}$ is in trans-position in comparison with the ethylenic double bond. In 6-12 the $\mathrm{Pt}(\mathrm{II})$ atom is surrounded by an aromatic $\mathrm{C}$ atom and the ethylenic $\mathrm{C}=\mathrm{C}$ double bond of the aryl olefin, and by $\mathrm{N}$ and $\mathrm{O}$ atoms of the 8-OquiN resulting in a slightly distorted square-planar coordination environment, the donor $\mathrm{N}$ is in cis-position_in comparison with the ethylenic double bond. Complex 7 exhibits high activities on human cancer cells $\mathrm{KB}$, Hep-G2, Lu and MCF-7 with $\mathrm{IC}_{50}$ values of 4.2, 2.7, 4.3 and $3.6 \mu \mathrm{M}$, respectively.
\end{abstract}

Keywords: Platinum(II) complex, Organoplatinum, Safrole, Eugenol, 8-Hydroxyquinolines.

\section{Introduction}

Recent years have witnessed an unprecedented progress in biological applications of metal coordination compounds of biologically active ligands because of their key role in clinical 
therapy. Platinum complexes are particularly suitable for this purpose. Interest in platinum complexes containing biological active natural compounds is increasing. Complexes of platinum(II) with caffeine [1],_ $\beta$-carboline alkaloids [2], theophylline [3], oleanoic acid [4], propyl gallate [5], hydroxyquinolines [6,7] and so forth, were synthesized and their antitumor and antiviral activities have been demonstrated.

Our idea was to design new organoplatinum(II) anticancer compounds containing aryl olefins which already exert biological properties expecting synergistic effects. Thus, for the first time, methyleugenol (4-allyl-1,2-dimethoxybenzene, from a number of plants such as Cinnamomum cordatum, lemongrass, star anise...), safrole (4-allyl-1,2-methylendioxybenzene, the main component of sassafras oil), eugenol (4-allyl-2-methoxyphenol, a phenolic major component of a number of medicinal plants such as clove and tulsi), and some alkyl eugenoxyacetates were introduced into the coordination entity of platinum(II) $[8,9,10,11$, respectively], then the obtained key compounds were transformed into organoplatinum complexes comprising these aryl olefins or chelating aryl olefins and various amines. It was shown that many synthesized complexes exhibit impressive high in vitro cytotoxicityon four human cancer cell lines KB, Hep-G2, Lu, MCF7 with $\mathrm{IC}_{50}=0.4-9.6 \mu \mathrm{M}$. Some complexes gave better antitumor activity than cisplatin against examined cell lines [10, $12-15]$.

In the current study we have continued this line of research, focusing on the synthesis, characterization of a series of organoplatinum(II) complexes containing safrole (Saf) as well as methyl eugenoxyacetate (Meteug) or ethyl eugenoxyacetate (Eteug), all of them differing in the bidentate quinolin-8-olates (OquiN). In addition, several 8-hydroxyquinolines are already known for their antimicrobial and antiprotozoal activity and many of them are or were used in clinical practice [16]. Their biological activity is often ascribed to their ability to bind di- and trivalent transition metal ions such as copper(II), zinc(II), and iron(III) [17].

\section{Experimental}

\subsection{General and instrumentation}

Elemental analysis: Pt was analyzed according to the weight method [18], $\mathrm{C}$ and $\mathrm{H}$ were analyzed on a LECO CHNS model 932 elemental analyzer. The IR spectra were recorded on an IMPACK-410 NICOLET spectrometer in $\mathrm{KBr}$ discs in the range $400-4000 \mathrm{~cm}^{-1}$. The NMR spectra were recorded on a Bruker AVANCE $500 \mathrm{MHz}$, all at 298-300 K, with TMS as the internal standard in suitable solvents (Tables 2-3). MS spectra were recorded on a LCMSD-Trap-SL instrument. The in vitro cytotoxicity were tested at the Experimental Biological Laboratory - Institute of Chemistry of Natural Compounds (in Hanoi), according 
to the method described in reference [19], $\mathrm{IC}_{50}$ values were calculated based on OD values taken on an Elisa instrument at 515-540 nm.

\subsection{X-ray diffraction analysis}

Single-crystal X-ray diffraction were collected at $293 \mathrm{~K}$ on an Agilent Super Nova diffractometer equipped with an Eos CCD detector, using MoK $\alpha$ radiation $(\lambda=0.71073 \AA)$. The images were interpreted and integrated with CrysAlisPRO [20] and the implemented absorption correction was applied. Using Olex2 [21], the structure was solved with the ShelXS [22] structure solution program using Direct Methods and refined with the ShelXL [23] refinement package using full-matrix least squares minimization on $\mathrm{F}^{2}$. Non-hydrogen atoms were refined anisotropically. Hydrogen atoms were located in the calculated positions. A summary of crystal data and refinement details is given in Table 1.

Table 1. Crystal data and refinement details for crystal structures of complex 9.

\begin{tabular}{|l|l|l|l|}
\hline Formula & $\mathrm{C}_{24} \mathrm{H}_{25} \mathrm{NO}_{5} \mathrm{Pt}$ & $V\left(\AA^{3}\right)$ & $1061.78(5)$ \\
\hline Formula weight & 602.54 & $Z$ & 2 \\
\hline Crystal size $(\mathrm{mm})$ & $0.25 \times 0.2 \times 0.2$ & $\rho_{\text {calc }}\left(\mathrm{g} / \mathrm{cm}^{3}\right)$ & 1.885 \\
\hline Radiation & $\mathrm{Mo} \mathrm{K \alpha}(\lambda=0.71073)$ & $\mu\left(\mathrm{mm}^{-1}\right)$ & 6.644 \\
\hline Temperature $(\mathrm{K})$ & $293.4(6)$ & $F(000)$ & 588.0 \\
\hline Crystal system & triclinic & $2 \theta$ value $\left(^{\circ}\right)$ & $5.222-52.763$ \\
\hline Space group & $P-1$ & Reflections collected & 21675 \\
\hline$a(\AA)$ & $9.3398(3)$ & Independent reflections & 4316 \\
\hline$b(\AA)$ & $10.3892(3)$ & $R_{\text {int }}$ & 0.0376 \\
\hline$c(\AA)$ & $11.4404(3)$ & Data/restraints/parameters & $4316 / 0 / 283$ \\
\hline$\alpha\left(^{\circ}\right)$ & $92.004(2)$ & $R\left[F^{2}>2 \sigma\left(F^{2}\right)\right], w R\left(F^{2}\right)$ & $0.0163,0.0365$ \\
\hline$\beta\left(^{\circ}\right)$ & $104.825(2)$ & Goodness-of-fit on $\mathrm{F}^{2}$ & 1.074 \\
\hline$\gamma\left(^{\circ}\right)$ & $97.310(2)$ & Largest diff. peak, hole $\left(\mathrm{e} \AA^{-3}\right)$ & $0.43,-0.58$ \\
\hline
\end{tabular}

\subsection{Preparation of complexes 1-5}

The preparation of $\mathrm{K}\left[\mathrm{Pt}(\mathrm{Saf}) \mathrm{Cl}_{3}\right], \mathrm{K}\left[\mathrm{Pt}(\mathrm{Meteug}) \mathrm{Cl}_{3}\right]$, (here 'Saf' and 'Meteug' are safrole and methyl eugenoxyacetate, respectively, Fig 1) were previously described in $[9,11]$.

To a solution of $\mathrm{K}\left[\mathrm{Pt}(\mathrm{Saf}) \mathrm{Cl}_{3}\right](201 \mathrm{mg}, 0.4 \mathrm{mmol})$ or $\mathrm{K}\left[\mathrm{Pt}(\mathrm{Meteug}) \mathrm{Cl}_{3}\right]$ (208 mg, 0.4 $\mathrm{mmol})$ in $15 \mathrm{~mL}$ ethanol/water (4:1 by volume) an 8 -hydroxyquinoline $(0.4 \mathrm{mmol})$ in_15 $\mathrm{mL}$ ethanol or ethanol/water (4:1 by volume) was slowly added and stirred at room temperature for 1 - 2 hours. The mixture was then left at room temperature or refrigerated for several hours. The resulting precipitate was collected, washed with ethanol/water (1:1 by volume) and dried. 
2.3.1. [PtCl(Saf)(2-methyl-8-O-quinoline)] (1, ( $\eta^{2}-5$-allyl-1,3-benzodioxole)chlorido(2methylquinolin-8-olato- $\kappa^{2} N, O$ )-platinum(II))

The yield was $159 \mathrm{mg}$ (72\%), yellow powder. Anal. Calc. For $\left[\mathrm{C}_{20} \mathrm{H}_{18} \mathrm{ClNO}_{3} \mathrm{Pt}\right]: \mathrm{C}, 43.60 ; \mathrm{H}$, 3.29; Pt, 35.41. Found: 43.28; H, 3.48; Pt, 35.70. IR (cm-1): 3057, 2976, 2857 (vCH); 1600, 1553, $1490(v \mathrm{C}=\mathrm{C} / \mathrm{C}=\mathrm{N})$. For ${ }^{1} \mathrm{H}$ NMR see Table 2 .

\subsection{2. [PtCl(Saf)(5,7-dichloro-8-O-quinoline)] (2, ( $\eta^{2}-5$-allyl-1,3-benzodioxole)chlorido-} (5,7-dichloroquinolin-8-olato- $\left.\kappa^{2} N, O\right)$-platinum(II))

The yield was $181 \mathrm{mg}(75 \%)$, yellow powder. Anal. Calc. For $\left[\mathrm{C}_{19} \mathrm{H}_{14} \mathrm{Cl}_{3} \mathrm{NO}_{3} \mathrm{Pt}\right]: \mathrm{C}, 37.67 ; \mathrm{H}$, 2.33; Pt, 32.20. Found: C, 37.35; H, 2.53; Pt, 32.45. IR ( $\left.\mathrm{cm}^{-1}\right)$ : 3050, 2980, 2899 (vCH); 1595, 1563, $1493(v \mathrm{C}=\mathrm{C} / \mathrm{C}=\mathrm{N})$. For ${ }^{1} \mathrm{H}$ NMR see Table 2.

\subsection{3. [PtCl(Saf)(5,7-dichloro-2-methyl-8-O-quinoline)] (3, $\left(\eta^{2}-5\right.$-allyl-1,3-}

benzodioxole)chlorido(5,7-dichloro-2-methylquinolin-8-olato- $\kappa^{2} \mathrm{~N}, \mathrm{O}$ )-platinum(II))

The yield was $173 \mathrm{mg}(70 \%)$, yellow powder. Anal. Calc. For $\left[\mathrm{C}_{20} \mathrm{H}_{16} \mathrm{Cl}_{3} \mathrm{NO}_{3} \mathrm{Pt}\right]$ : C, 38.76; $\mathrm{H}$, 2.60; Pt, 31.48. Found: C, 38.47; H, 2.82; Pt, 31.75. IR (cm-1): 3060, 2985, 2898 (vCH); 1600, 1554, $1501(v \mathrm{C}=\mathrm{C} / \mathrm{C}=\mathrm{N})$. For ${ }^{1} \mathrm{H}$ NMR see Table 2. ESI-MS-MS: $653.7 / 100 \%\left([\mathrm{M}+\mathrm{Cl}]^{-}\right)$; $+\mathrm{MS}: 583.9 / 100 \%\left([\mathrm{M}-\mathrm{Cl}]^{+}\right), 620.5 / 15 \%\left([\mathrm{M}+\mathrm{H}]^{+}\right)$.

Table 2. ${ }^{1} \mathrm{H}$ NMR signals of complexes 1-3 and 6-8, $\delta(\mathrm{ppm}), J(\mathrm{~Hz})$.

\begin{tabular}{|c|c|c|c|c|c|c|}
\hline & $\mathbf{1} / \mathrm{CDCl}_{3}$ & $2 / \mathrm{CDCl}_{3}$ & $3 / \mathrm{CDCl}_{3}$ & 6/CDCl ${ }_{3}$ & $7 / \mathrm{CDCl}_{3}$ & $8 / \mathrm{CDCl}_{3}$ \\
\hline H1a & $5.91 \mathrm{~s}$ & $5.91 \mathrm{~s}$ & $5.91 \mathrm{~s}$ & $5.84 \mathrm{~d} ;{ }^{2} J 1$ & $5.85 \mathrm{~d} ;{ }^{2} J 1$ & $5.86 \mathrm{~d} ;{ }^{2} J 1$ \\
\hline H1a' & $5.91 \mathrm{~s}$ & $5.91 \mathrm{~s}$ & $5.91 \mathrm{~s}$ & $5.87 \mathrm{~d} ;{ }^{2} J 1$ & $5.88 \mathrm{~d} ;{ }^{2} J 1$ & $5.89 \mathrm{~d} ;{ }^{2} J 1$ \\
\hline $\mathrm{H} 3$ & $6.84 \mathrm{~s}$ & $6.83 \mathrm{~d} ;{ }^{4} J 1.5$ & $6.83 \mathrm{~s}$ & $6.61 \mathrm{~s}$ & $6.61 \mathrm{~s}$ & $6.60 \mathrm{~s}$ \\
\hline H5 & $6.79 \mathrm{~d} ; 3^{3} J 7.5$ & $\begin{array}{c}6.78 \mathrm{dd} ; \\
{ }^{3} J 8 ;{ }^{4} J 1.5\end{array}$ & $6.78 \mathrm{~d} ;{ }^{3} J 7.5$ & - & & - \\
\hline H6 & $6.72 \mathrm{~d} ; 3^{3} J 7.5$ & $6.71 \mathrm{~d} ;{ }^{3} J 8$ & $6.72 \mathrm{~d} ;{ }^{3} J 7.5$ & $7.23 \mathrm{~s}$ & $\begin{array}{c}7.18 \mathrm{~s} \\
{ }^{3} J_{\mathrm{PtH}} 35\end{array}$ & $\begin{array}{c}7.27 \mathrm{~s} \\
{ }^{3} J_{\mathrm{PtH}} \mathrm{OV} .\end{array}$ \\
\hline $\mathrm{H} 7 \mathrm{a}$ & $3.21 \mathrm{br}$. & $\begin{array}{c}3.33 \mathrm{dd} ; \\
{ }^{2} J 15 ;{ }^{3} J 8\end{array}$ & $\begin{array}{l}3.22 \mathrm{dd} ; \\
2 J 15 ;{ }^{2} J 7\end{array}$ & $\begin{array}{c}2.82 \mathrm{~d} ;{ }^{2} J 17 \\
{ }^{3} J_{\mathrm{PtH}} 95\end{array}$ & $\begin{array}{c}2.85 \mathrm{~d} ;{ }^{2} J 17 \\
{ }^{3} J_{\mathrm{PtH}} 95\end{array}$ & $\begin{array}{c}2.81 \mathrm{~d} ;{ }^{2} J 17 \\
{ }^{3} J_{\mathrm{PtH}} 95\end{array}$ \\
\hline $\mathrm{H} 7 \mathrm{~b}$ & $3.57 \mathrm{br}$. & $\begin{array}{c}3.63 \mathrm{dd} ; \\
{ }^{2} J 15 ;{ }^{3} J 6\end{array}$ & $\begin{array}{c}3.56 \mathrm{~d} ; \\
{ }^{2} J 15\end{array}$ & $\begin{array}{c}3.74 \mathrm{dd} ; \\
2 J 17 ; 3 \mathrm{~J} 6.5\end{array}$ & $\begin{array}{c}3.69 \mathrm{dd} ; \\
{ }^{2} J 17 ; 3 \mathrm{~J} 6\end{array}$ & $\begin{array}{c}3.71 \mathrm{dd} ; \\
{ }^{2} J 17 ; 3 \mathrm{~J} 6\end{array}$ \\
\hline H8 & $\begin{array}{l}5.65 \mathrm{~m} ; \\
{ }^{2} J_{\mathrm{PtH}} 70\end{array}$ & $\begin{array}{l}5.75 \mathrm{~m} ; \\
{ }^{2} J_{\mathrm{PtH}} 70\end{array}$ & $\begin{array}{l}5.73 \mathrm{~m} ; \\
{ }^{2} J_{\mathrm{PtH}} 70\end{array}$ & $\begin{array}{l}4.98 \mathrm{~m} ; \\
{ }^{2} J_{\mathrm{PtH}} 75\end{array}$ & $\begin{array}{l}4.78 \mathrm{~m} ; \\
{ }^{2} J_{\mathrm{PtH}} 75\end{array}$ & $\begin{array}{l}5.01 \mathrm{~m} ; \\
{ }^{2} J_{\mathrm{PtH}} 75\end{array}$ \\
\hline H9cis & $\begin{array}{c}4.78 \mathrm{~d} ;{ }^{3} J 7 ; \\
{ }^{2} J_{\mathrm{PtH}} 70\end{array}$ & $\begin{array}{c}4.92 \mathrm{~d} ;{ }^{3} J 8 ; \\
{ }^{2} J_{\mathrm{PtH}} 70\end{array}$ & $\begin{array}{c}4.87 \mathrm{~d} ;{ }^{3} J 8 \\
{ }^{2} J_{\mathrm{PtH}} 70\end{array}$ & $\begin{array}{c}4.35 \mathrm{~d} ;{ }^{3} J 7.5 ; \\
{ }^{2} J_{\mathrm{PtH}} 75\end{array}$ & $\begin{array}{c}4.07 \mathrm{~d} ;{ }^{3} J 8 ; \\
{ }^{2} J_{\mathrm{PtH}} 75\end{array}$ & $\begin{array}{c}4.38 \mathrm{~d} ;{ }^{3} J 8 ; \\
{ }^{2} J_{\mathrm{PtH}} 75\end{array}$ \\
\hline H9trans & $\begin{array}{c}4.75 \mathrm{~d} ;{ }^{3} J 14 ; \\
{ }^{2} J_{\mathrm{PtH}} 70\end{array}$ & $\begin{array}{c}4.88 \mathrm{~d} ;{ }^{3} J 15 ; \\
{ }^{2} J_{\mathrm{PtH}} 70\end{array}$ & $\begin{array}{c}4.86 \mathrm{~d} ;{ }^{3} J 13 ; \\
{ }^{2} J_{\mathrm{PtH}} 70\end{array}$ & $\begin{array}{c}3.72 \mathrm{~d} ;{ }^{3} J 13 ; \\
{ }^{2} J_{\mathrm{PtH}} 75\end{array}$ & $\begin{array}{c}3.93 \mathrm{~d} ;{ }^{3} J 13 \\
{ }^{2} J_{\mathrm{PtH}} 75\end{array}$ & $\begin{array}{c}3.83 \mathrm{~d} ;{ }^{3} J 13 \\
{ }^{2} J_{\mathrm{PtH}} 75\end{array}$ \\
\hline H12 & - & $\begin{array}{c}9.16 \mathrm{dd} ; \\
{ }^{3} J 5.5 ;{ }^{4} J 1.5\end{array}$ & - & - & $8.15 \mathrm{~d} ;{ }^{3} J 5$ & - \\
\hline
\end{tabular}




\begin{tabular}{|c|c|c|c|c|c|c|}
\hline H12a & $3.17 \mathrm{~s}$ & - & $3.19 \mathrm{~s}$ & $2.67 \mathrm{~s}$ & - & $2.69 \mathrm{~s}$ \\
\hline $\mathrm{H} 13$ & $7.31 \mathrm{~d} ;{ }^{3} J 8.5$ & $\begin{array}{c}7.68 \mathrm{dd} ; \\
{ }^{3} J 8.5 ;{ }^{3} J 5.5\end{array}$ & $\begin{array}{c}7.42 \mathrm{~d} ; \\
3\end{array} 8.5$ & $7.24 \mathrm{~d} ;{ }^{3} J 8.5$ & $\begin{array}{c}7.56 \mathrm{dd} ;{ }^{3} J \\
8.5 ;{ }^{3} J 5\end{array}$ & $7.33 \mathrm{~d} ;{ }^{3} J 8.5$ \\
\hline H14 & $8.22 \mathrm{~d} ;{ }^{3} J 8.5$ & $\begin{array}{c}8.75 \mathrm{dd} ;{ }^{3} J \\
8.5 ;{ }^{4} J 1.5\end{array}$ & $\begin{array}{c}8.56 \mathrm{~d} ; \\
{ }^{3} J 8.5 ;\end{array}$ & $8.15 \mathrm{~d} ;{ }^{3} J 8.5$ & $8.66 \mathrm{~d} ;{ }^{3} J 8.5$ & $8.49 \mathrm{~d} ;{ }^{3} J 8.5$ \\
\hline H15 & $6.96 \mathrm{~d} ;{ }^{3} J 7.5$ & - & - & $7.20 \mathrm{~d} ;{ }^{3} J 8$ & - & - \\
\hline H16 & $7.33 \mathrm{t} ;{ }^{3} J 7.5$ & $7.64 \mathrm{~s}$ & $7.54 \mathrm{~s}$ & $7.46 \mathrm{t} ;{ }^{3} J 8$ & $7.74 \mathrm{~s}$ & $7.65 \mathrm{~s}$ \\
\hline H17 & $6.96 \mathrm{~d} ;{ }^{3} J 7.5$ & - & - & $7.00 \mathrm{~d} ;{ }^{3} J 8$ & - & - \\
\hline
\end{tabular}

2.3.4. [PtCl(Meteug)(5,7-dichloro-8-O-quinoline)] (4, chlorido(5,7-dichloroquinolin-8-

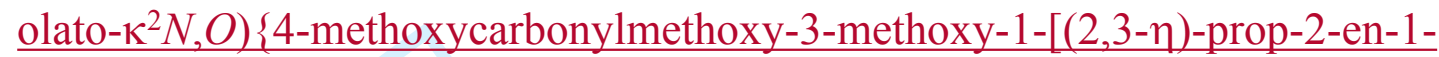
yl)benzene ; platinum(II))

The yield was $207 \mathrm{mg}$ (76\%), yellow powder. Anal. Calc. For $\left[\mathrm{C}_{22} \mathrm{H}_{20} \mathrm{Cl}_{3} \mathrm{NO}_{5} \mathrm{Pt}\right]: \mathrm{C}, 38.87 ; \mathrm{H}$, 2.97; Pt, 28.69. Found: C, 39.05; H, 3.15; Pt, 28.37. IR ( $\left.\mathrm{cm}^{-1}\right): 3080,3005$ 2952, 2828 (vCH); $1731(v \mathrm{C}=\mathrm{O}) ; 1593,1564,1515(v \mathrm{C}=\mathrm{C} / \mathrm{C}=\mathrm{N})$. For ${ }^{1} \mathrm{H}$ NMR see Table 3.-ESI-MS-MS: $713.8 / 40 \%\left([\mathrm{M}+\mathrm{Cl}]^{-}\right) ;=\mathrm{MS}: 643.9 / 100 \%\left([\mathrm{M}-\mathrm{Cl}]^{+}\right), 680.7 / 50 \%\left([\mathrm{M}+\mathrm{H}]^{+}\right)$.

2.3.5. [PtCl(Meteug)(5,7-dichloro-2-methyl-8-O-quinoline)] (5, chlorido(5,7-dichloro-2-

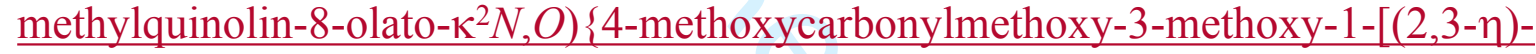
prop-2-en-1-yl)benzene platinum(II))

The yield was $180 \mathrm{mg}(65 \%)$, yellow powder. Anal. Calc. For $\left[\mathrm{C}_{23} \mathrm{H}_{22} \mathrm{Cl}_{3} \mathrm{NO}_{5} \mathrm{Pt}\right]$ : C, 39.81; $\mathrm{H}$, 3.20; Pt, 28.11. Found: C, 40.12; H, 3.05; Pt, 28.27. IR ( $\left.\mathrm{cm}^{-1}\right)$ : 3076, 2953, 2858 (vCH); 1757 $(v \mathrm{C}=\mathrm{O}) ; 1595,1586,1554,1514(v \mathrm{C}=\mathrm{C} / \mathrm{C}=\mathrm{N})$. For ${ }^{1} \mathrm{H}$ NMR see Table 3. -MSESI-MS: 727.8/100\% $\left([\mathrm{M}+\mathrm{Cl}]^{-}\right) ; \neq \mathrm{MS}: 657.9 / 100 \%\left([\mathrm{M}-\mathrm{Cl}]^{+}\right), 693.8 / 30 \%\left([\mathrm{M}+\mathrm{H}]^{+}\right)$.

\subsection{Preparation of complexes 6-12}

The preparation of $\left[\mathrm{Pt}_{2} \mathrm{Cl}_{2}(\mathrm{Saf}-1 \mathrm{H})_{2}\right]$ and $\left[\mathrm{Pt}_{2} \mathrm{Cl}_{2}(\text { Eteug- } 1 \mathrm{H})_{2}\right]$ ('Saf- $1 \mathrm{H}$ ' and 'Eteug- $1 \mathrm{H}$ ' are deprotonated safrole and deprotonated ethyl eugenoxyacetate, respectively, Fig 1) were previously described in $[9,11]$.

To a mixture of $\left[\mathrm{Pt}_{2} \mathrm{Cl}_{2}(\mathrm{Saf}-1 \mathrm{H})_{2}\right](157 \mathrm{mg}, 0.2 \mathrm{mmol})$ or $\left[\mathrm{Pt}_{2} \mathrm{Cl}_{2}(\text { Eteug-1H })_{2}\right](\underline{192 \mathrm{mg}, 0.2}$ mmol) in $12 \mathrm{~mL}$ acetone/ethanol (5:1 by volume) an 8 -hydroxyquinoline ( $0.4 \mathrm{mmol})$ in_10 $\mathrm{mL}$ acetone or acetone/ethanol (5:1 by volume) was slowly added and stirred at room temperature for 1 - 2 hours. The resulting solution was kept at room temperature for several hours. The precipitate was collected, washed with ethanol and dried. 


\subsection{1. [Pt(Saf-1H)(2-methyl-8-O-quinoline $)]_{-}\left(6_{2}\left(\eta^{2}-5-\right.\right.$ allyl-1,3-benzodioxole- $\left.\kappa C^{6}\right)(2-$}

$\underline{\left.\text { methylquinolin-8-olato- } \kappa^{2} N, O\right) \text {-platinum(II) }}$

The yield was $154 \mathrm{mg}$ (75\%), yellow powder. Anal. Calc. For $\left[\mathrm{C}_{20} \mathrm{H}_{17} \mathrm{NO}_{3} \mathrm{Pt}\right]$ : C, 46.70; $\mathrm{H}$, 3.33; Pt, 37.92. Found: C, 46.42; H, 3.12; Pt, 38.21. IR ( $\left.\mathrm{cm}^{-1}\right)$ : 2977, 2876, 2830 (vCH); 1598, 1564, $1507(v \mathrm{C}=\mathrm{C} / \mathrm{C}=\mathrm{N})$. For ${ }^{1} \mathrm{H}$ NMR see Table 2 .

\subsection{2. [Pt(Saf-1H)(5,7-dichloro-8-O-quinoline)] (7, ( $\eta^{2}-5$-allyl-1,3-benzodioxole-}

\section{$\underline{\left.\left.\kappa C^{6}\right)\left(5,7 \text {-dichloroquinolin-8-olato- } \kappa^{2} N, O\right) \text {-platinum(II) }\right)}$}

The yield was $155 \mathrm{mg}$ (68\%), dark yellow powder. Anal. Calc. For $\left[\mathrm{C}_{19} \mathrm{H}_{13} \mathrm{Cl}_{2} \mathrm{NO}_{3} \mathrm{Pt}\right]: \mathrm{C}$, 40.09; H, 2.30; Pt, 34.27. Found: C, 39.83; H, 2.52; Pt, 34.53. IR (cm-1): 3055, 2970, 2894 $(v \mathrm{CH}) ; 1588,1562,1496(v \mathrm{C}=\mathrm{C} / \mathrm{C}=\mathrm{N})$. For ${ }^{1} \mathrm{H}$ NMR see Table 2.

\subsection{3. [Pt(Saf-1H)(5,7-dichloro-2-methyl-8-O-quinoline $)]\left(8,\left(\eta^{2}-5\right.\right.$-allyl-1,3-}

\section{benzodioxole- $\left.\kappa C^{6}\right)\left(2\right.$-methyl-5,7-dichloroquinolin-8-olato- $\left.\kappa^{2} N, O\right)$-platinum(II))}

The yield was $168 \mathrm{mg}$ (72\%), yellow powder. Anal. Calc. For $\left[\mathrm{C}_{20} \mathrm{H}_{15} \mathrm{C}_{12} \mathrm{NO}_{3} \mathrm{Pt}\right]$ : $\mathrm{C}$, 41.18; H, 2.59; Pt, 33.44. Found: C, 39.90; H, 2.30; Pt, 33.72. IR ( $\left.\mathrm{cm}^{-1}\right): 3060,2978,2887$ $(\mathrm{vCH}) ; 1603,1582,1551,1502(v \mathrm{C}=\mathrm{C} / \mathrm{C}=\mathrm{N})$. For ${ }^{1} \mathrm{H}$ NMR see Table 2.-ESI-MS-MS: 619.8/100\% $\left([\mathrm{M}+\mathrm{Cl}]^{-}\right) ;+\mathrm{MS}: 583.9 / 100 \%\left([\mathrm{M}+\mathrm{H}]^{+}\right)$.

\subsection{4. [Pt(Eteug-1H)(2-methyl-8-O-quinoline)] (9, \{4-ethoxycarbonylmethoxy-3-}

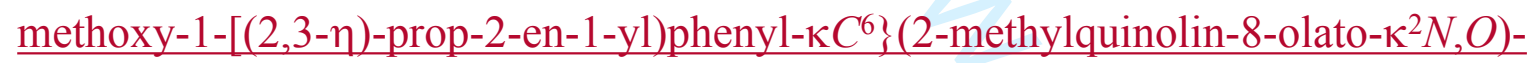
platinum(II))

The yield was $161 \mathrm{mg}(67 \%)$, yellow powder. Anal. Calc. For $\left[\mathrm{C}_{24} \mathrm{H}_{25} \mathrm{NO}_{5} \mathrm{Pt}\right]: \mathrm{C}, 47.84 ; \mathrm{H}$, 4.18; Pt, 32.38. Found: C, 48.15; H, 4.03; Pt, 32.12. IR ( $\left.\mathrm{cm}^{-1}\right): 3051,2944,2843$ (vCH); 1717 $(v \mathrm{C}=\mathrm{O}) ; 1557,1501,1459(v \mathrm{C}=\mathrm{C} / \mathrm{C}=\mathrm{N})$. For ${ }^{1} \mathrm{H}$ NMR see Table 3. Yellow crystals suitable for X-ray diffraction were obtained by slow evaporation within $24 \mathrm{~h}$ from a saturated acetoneethanol mixture (6:1 by volume) solution at room temperature.

Table 3. ${ }^{1} \mathrm{H}$ NMR signals of complexes 4, 5 and 9-12, $\delta(\mathrm{ppm}), J(\mathrm{~Hz})$.

\begin{tabular}{|c|c|c|c|c|c|c|}
\hline & $4 / \mathrm{CDCl}_{3}$ & $\mathbf{5} / \mathrm{CDCl}_{3}$ & $\mathbf{9} / \mathrm{Me}_{2} \mathrm{CO}$ & $\mathbf{1 0} / \mathrm{CDCl}_{3}$ & 11/ $\mathrm{Me}_{2} \mathrm{CO}$ & $12 / \mathrm{CDCl}_{3}$ \\
\hline \multirow[t]{2}{*}{ H1a } & \multirow{2}{*}{$4.63 \mathrm{~s}$} & \multirow{2}{*}{$4.63 \mathrm{~s}$} & $4.57 \mathrm{~d} ;{ }^{2} J 16$ & $4.76 \mathrm{~s}$ & $4.66 \mathrm{~s}$ & $4.78 \mathrm{~d} ; ;^{2} J 16$ \\
\hline & & & $4.58 \mathrm{~d} ;{ }^{2} J 16$ & $4.76 \mathrm{~s}$ & $4.66 \mathrm{~s}$ & $4.79 \mathrm{~d} ;{ }^{2} J 16$ \\
\hline \multirow[t]{2}{*}{ H1b } & \multirow{2}{*}{$3.77 \mathrm{~s}$} & \multirow{2}{*}{$3.77 \mathrm{~s}$} & $4.18 \mathrm{q} ; 3 J 7$ & $4.31 \mathrm{q} ;{ }^{3} J 7$ & $4.24 \mathrm{q} ;{ }^{3} J 7$ & $4.28 \mathrm{q} ; 3^{3} J 7$ \\
\hline & & & $4.19 \mathrm{q} ;{ }^{3} J 7$ & $4.32 \mathrm{q} ;{ }^{3} J 7$ & $4.26 \mathrm{q} ;{ }^{3} J 7$ & $4.29 \mathrm{q} ;{ }^{3} J 7$ \\
\hline H1c & - & - & $1.23 \mathrm{t} ;{ }^{3} \mathrm{~J} 7$ & $1.33 \mathrm{t} ;{ }^{3} \mathrm{~J} 7$ & $1.28 \mathrm{t} ;{ }^{3} \mathrm{~J} 7$ & $1.31 \mathrm{t} ;{ }^{3} J 7$ \\
\hline
\end{tabular}




\begin{tabular}{|c|c|c|c|c|c|c|}
\hline $\mathrm{H} 2 \mathrm{a}$ & $3.69 \mathrm{~s}$ & $3.66 \mathrm{~s}$ & $3.69 \mathrm{~s}$ & $3.84 \mathrm{~s}$ & $3.76 \mathrm{~s}$ & $3.83 \mathrm{~s}$ \\
\hline H3 & $6.90 \mathrm{~s}$ & $6.89 \mathrm{~s}$ & $6.67 \mathrm{~s}$ & $6.70 \mathrm{~s}$ & $6.74 \mathrm{~s}$ & $6.66 \mathrm{~s}$ \\
\hline H5 & $6.86 \mathrm{~d} ;{ }^{3} J 8$ & $6.86 \mathrm{~d} ;{ }^{3} J 8$ & - & - & - & - \\
\hline H6 & $6.75 \mathrm{~d} ;{ }^{3} J 8$ & $6.75 \mathrm{~d} ;{ }^{3} J 8$ & $\begin{array}{c}7.13 \mathrm{~s} ; \\
3 J_{\mathrm{PtH}} 35\end{array}$ & $\begin{array}{c}7.20 \mathrm{~s} \\
{ }^{3} J_{\mathrm{PtH}} 35\end{array}$ & $\begin{array}{c}7.11 \mathrm{~s} ; \\
{ }^{3} J_{\mathrm{PtH}} 35\end{array}$ & $\begin{array}{c}7.25 \mathrm{~s} ; \\
3^{3} J_{\mathrm{PtH}} 35\end{array}$ \\
\hline $\mathrm{H7a}$ & $\begin{array}{l}3.33 \mathrm{dd} ; \\
2 J 15 ; 3 J 7\end{array}$ & $\begin{array}{l}3.15 \mathrm{~m} ; \\
\text { ov. }\end{array}$ & $2.68 \mathrm{ov}$. & $\begin{array}{c}2.87 \mathrm{~d} ;{ }^{2} J 17 ; \\
{ }^{3} J_{\mathrm{PtH}} 95\end{array}$ & $\begin{array}{c}2.86 \mathrm{~d} ;{ }^{2} J 17 ; \\
{ }^{3} J_{\mathrm{PtH}} 95\end{array}$ & $\begin{array}{c}2.80 \mathrm{~d} ;{ }^{2} J 17 ; \\
{ }^{3} J_{\mathrm{PtH}} 95\end{array}$ \\
\hline $\mathrm{H} 7 \mathrm{~b}$ & $\begin{array}{l}3.63 \mathrm{dd} ; \\
2 J 15 ; 37\end{array}$ & $\begin{array}{l}3.62 \mathrm{~m} \\
\text { ov. }\end{array}$ & $\begin{array}{l}3.59 \mathrm{dd} ; \\
2 J 7 ; 3 J 6\end{array}$ & $\begin{array}{l}3.79 \mathrm{dd} ; \\
2 J 17 ;^{3} J 6\end{array}$ & $\begin{array}{l}3.67 \mathrm{dd} \\
2 J 73^{3} J 6\end{array}$ & $\begin{array}{l}3.73 \mathrm{dd} ; \\
2 J 17 ; 3 \mathrm{~J} 6\end{array}$ \\
\hline $\mathrm{H} 8$ & $\begin{array}{l}5.78 \mathrm{~m} ; \\
{ }^{2} J_{\mathrm{PtH}} 70\end{array}$ & $\begin{array}{l}5.77 \mathrm{~m} ; \\
{ }^{2} J_{\mathrm{PtH}} 70\end{array}$ & $\begin{array}{l}5.02 \mathrm{~m} ; \\
{ }^{2} J_{\mathrm{PtH}} 75\end{array}$ & $\begin{array}{l}5.03 \mathrm{~m} ; \\
{ }^{2} J_{\mathrm{PtH}} 75\end{array}$ & $\begin{array}{l}5.01 \mathrm{~m} ; \\
{ }^{2} J_{\mathrm{PtH}} 75\end{array}$ & $\begin{array}{l}5.00 \mathrm{~m} \\
2 J_{\mathrm{PtH}} 75\end{array}$ \\
\hline H9cis & $\begin{array}{c}4.92 \mathrm{~d} ;{ }^{3} J 8 \\
{ }^{2} J_{\mathrm{PtH}} 70\end{array}$ & $\begin{array}{c}4.89 \mathrm{~d} ;{ }^{3} J 8 \\
{ }^{2} J_{\mathrm{PtH}} 70\end{array}$ & $\begin{array}{c}4.44 \mathrm{~d} ;{ }^{3} J 7.5 ; \\
{ }^{2} J_{\mathrm{PtH}} 75\end{array}$ & $\begin{array}{c}4.27 \mathrm{~d} ;{ }^{3} J 6.5 ; \\
{ }^{2} J_{\mathrm{PtH}} 75\end{array}$ & $\begin{array}{c}4.36 \mathrm{~d} ;{ }^{3} J 7.5 \\
{ }^{2} J_{\mathrm{PtH}} 75\end{array}$ & $\begin{array}{l}4.40 \mathrm{~d} \\
3 \mathrm{~J} 7.5\end{array}$ \\
\hline H9trans & $\begin{array}{c}4.89 \mathrm{~d} ;{ }^{3} J 14 \\
{ }^{2} J_{\mathrm{PtH}} 70\end{array}$ & $\begin{array}{c}4.87 \mathrm{~d} ;{ }^{3} J 14 \\
{ }^{2} J_{\mathrm{PtH}} 70\end{array}$ & $\begin{array}{c}3.53 \mathrm{~d} ;{ }^{3} J 13 \\
{ }^{2} J_{\mathrm{PtH}} 75\end{array}$ & $\begin{array}{l}3.83 \mathrm{~d} \\
3 J 13.5\end{array}$ & $\begin{array}{c}3.83 \mathrm{~d} ;{ }^{3} J 13.5 \\
{ }^{2} J_{\mathrm{PtH}} 75\end{array}$ & $\begin{array}{l}3.85 \mathrm{~d} ; \\
3 J 14\end{array}$ \\
\hline $\mathrm{H} 12$ & $9.14 \mathrm{~d} ;{ }^{3} J 5$ & - & - & - & $8.62 \mathrm{~d} ;{ }^{3} J 5$ & - \\
\hline $\mathrm{H} 12 \mathrm{a}$ & - & $3.17 \mathrm{~s}$ & $2.68 \mathrm{~s}$ & $9.81 \mathrm{~s}$ & - & $2.69 \mathrm{~s}$ \\
\hline H13 & $\begin{array}{c}7.68 \mathrm{dd} ; \\
{ }^{3} J 8.5 ;{ }^{3} J 5\end{array}$ & $\begin{array}{l}7.42 \mathrm{~d} ; \\
3 J 8.5\end{array}$ & $\begin{array}{l}7.42 \mathrm{~d} ; \\
3 J 8.5\end{array}$ & $\begin{array}{l}8.01 \mathrm{~d} ; \\
3 J 8.5\end{array}$ & $\begin{array}{c}7.84 \mathrm{dd} ; \\
{ }^{3} J 8.5 ;{ }^{3} J 5\end{array}$ & $\begin{array}{l}7.35 \mathrm{~d} ; \\
3 J 8.5\end{array}$ \\
\hline H14 & $\begin{array}{l}8.75 \mathrm{~d} ; \\
{ }^{3} J 8.5\end{array}$ & $\begin{array}{l}8.56 \mathrm{~d} ; \\
{ }^{3} J 8.5\end{array}$ & $\begin{array}{l}8.29 \mathrm{~d} ; \\
{ }^{3} J 8.5\end{array}$ & $\begin{array}{l}8.43 \mathrm{~d} ; \\
3 J 8.5\end{array}$ & $\begin{array}{l}8.75 \mathrm{~d} ; \\
3 J 8.5\end{array}$ & $\begin{array}{l}8.49 \mathrm{~d} ; \\
3 J 8.5\end{array}$ \\
\hline H15 & - & - & $\begin{array}{l}7.01 \mathrm{dd} ; \\
{ }^{3} J 8 ;{ }^{4} J 1\end{array}$ & $\begin{array}{c}7.12 \mathrm{~d} ; \\
{ }^{3} \mathrm{~J} 8\end{array}$ & - & - \\
\hline H16 & $7.64 \mathrm{~s}$ & $7.54 \mathrm{~s}$ & $\begin{array}{c}7.38 \mathrm{t} \\
{ }^{3} \mathrm{~J} 8\end{array}$ & $\begin{array}{c}7.69 \mathrm{t} ; \\
3 \mathrm{~J} 8\end{array}$ & $7.81 \mathrm{~s}$ & $7.66 \mathrm{~s}$ \\
\hline H17 & - & - & $\begin{array}{c}6.98 \mathrm{dd} ; \\
{ }^{3} J 7.5 ;{ }^{4} \mathrm{~J} 1\end{array}$ & $\begin{array}{c}6.29 \mathrm{dd} ; \\
{ }^{3} J 7.5 ;{ }^{4} J 1\end{array}$ & - & - \\
\hline
\end{tabular}

\subsection{5. [Pt(Eteug-1H)(2-formyl-8-O-quinoline)] (10, \{4-ethoxycarbonylmethoxy-3-}

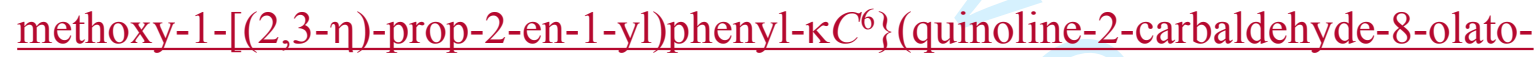
$\underline{\left.\kappa^{2} N, O\right) \text {-platinum(II)) }}$

The yield was $158 \mathrm{mg}(64 \%)$, brown powder. Anal. Calc. For $\left[\mathrm{C}_{24} \mathrm{H}_{23} \mathrm{NO}_{6} \mathrm{Pt}\right]: \mathrm{C}, 46.76$; H, 3.76; Pt, 31.64. Found: C, 47.02; H, 3.51; Pt, 31.93. IR ( $\left.\mathrm{cm}^{-1}\right): 3055,2931,2840(v \mathrm{CH})$; 1755, $1697(v \mathrm{C}=\mathrm{O}) ; 1589,1555,1454(v \mathrm{C}=\mathrm{C} / \mathrm{C}=\mathrm{N})$. For ${ }^{1} \mathrm{H}$ NMR see Table 3. MSESIMS: $650.9 / 100 \%\left([\mathrm{M}+\mathrm{Cl}]^{-}\right) ; \neq \mathrm{MS}: 616.9 / 80 \%\left([\mathrm{M}+\mathrm{H}]^{+}\right)$.

\subsection{6. [Pt(Eteug-1H)(5,7-dichloro-8-O-quinoline)] (11, \{4-ethoxycarbonylmethoxy-3-}

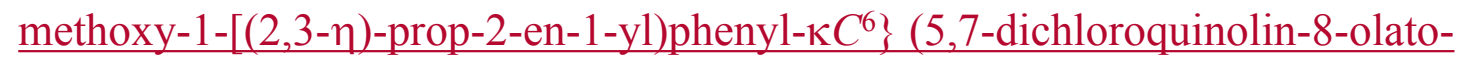
$\underline{\left.\kappa^{2} N, O\right) \text {-platinum(II)) }}$

The yield was $195 \mathrm{mg}$ (74\%), brown powder. Anal. Calc. For $\left[\mathrm{C}_{23} \mathrm{H}_{21} \mathrm{Cl}_{2} \mathrm{NO}_{5} \mathrm{Pt}\right]$ : C, 42.02; $\mathrm{H}$, 3.22; Pt, 29.67. Found: C, 42.30; H, 3.01; Pt, 29.89. IR $\left(\mathrm{cm}^{-1}\right): 3080,2919,2837(\mathrm{vCH})$; $1748(v \mathrm{C}=\mathrm{O}) ; 1562,1483,1443(v \mathrm{C}=\mathrm{C} / \mathrm{C}=\mathrm{N})$. For ${ }^{1} \mathrm{H}$ NMR see Table 3. 


\subsection{7. [Pt(Eteug-1H)(5,7-dichloro-2-methyl-8-O-quinoline)] $(12,\{4-$}

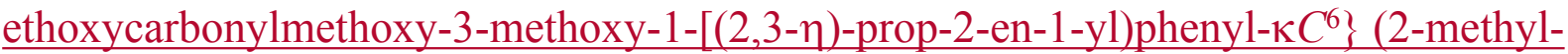

\section{5,7-dichloroquinolin-8-olato- $\kappa^{2} N, O$ )-platinum(II))}

The yield was $175 \mathrm{mg}(65 \%)$, yellow powder. Anal. Calc. For $\left[\mathrm{C}_{24} \mathrm{H}_{23} \mathrm{Cl}_{2} \mathrm{NO}_{5} \mathrm{Pt}\right]: \mathrm{C}, 42.93 ; \mathrm{H}$, 3.45; Pt, 29.05. Found: C, 43.18; H, 3.20; Pt, 28.81. IR ( $\left.\mathrm{cm}^{-1}\right)$ : 3065, 2984, 2933, 2835 (vCH); $1755(v \mathrm{C}=\mathrm{O}) ; 1590,1573,1548,1499(v \mathrm{C}=\mathrm{C} / \mathrm{C}=\mathrm{N})$. For ${ }^{1} \mathrm{H}$ NMR see Table 3. -MSESIMS: $705.9 / 100 \%\left([\mathrm{M}+\mathrm{Cl}]^{-}\right) ;+\mathrm{MS}: 671.9 / 100 \%\left([\mathrm{M}+\mathrm{H}]^{+}\right)$.

\section{Results and discussion}

Four previously prepared $[9,11]$ organoplatinum(II) complexes used for the synthesis of the title complexes are $\mathrm{K}\left[\mathrm{Pt}(\mathrm{Saf}) \mathrm{Cl}_{3}\right], \mathrm{K}\left[\mathrm{Pt}(\mathrm{Meteug}) \mathrm{Cl}_{3}\right],\left[\mathrm{Pt}_{2} \mathrm{Cl}_{2}(\mathrm{Saf}-1 \mathrm{H})_{2}\right]$ and $\left[\mathrm{Pt}_{2} \mathrm{Cl}_{2}\right.$ (Eteug$\left.1 \mathrm{H})_{2}\right]$, here 'Saf-1H' and 'Eteug-1H' are deprotonated safrole and deprotonated ethyl eugenoxyacetate, respectively. Their structures are presented in Fig. 1.<smiles></smiles>

$\mathrm{K}\left[\mathrm{PtCl}_{3}(\mathrm{Saf})\right]$<smiles>[X]CCP(C)(C)(C)CCc1ccc(OCC(=O)OC)c(OC)c1</smiles>

$\mathrm{K}\left[\mathrm{PtCl}_{3}(\right.$ Meteug $\left.)\right]$

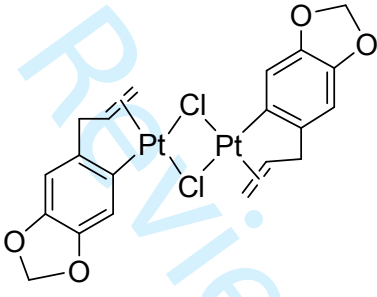

$\left[\mathrm{Pt}_{2} \mathrm{Cl}_{2}(\mathrm{Saf}-1 \mathrm{H})_{2}\right]$

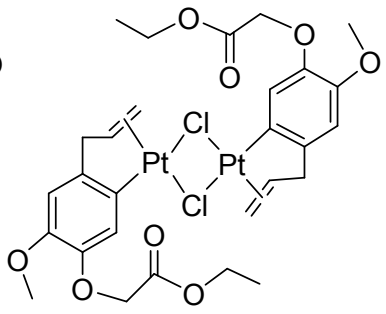

$\left[\mathrm{Pt}_{2} \mathrm{Cl}_{2}(\text { Eteug- } 1 \mathrm{H})_{2}\right]$

Figure 1. Starting organoplatinum(II) complexes.

\subsection{Synthesis and structure of complexes 1-5}

Treatment of $\mathrm{K}\left[\mathrm{Pt}(\mathrm{Saf}) \mathrm{Cl}_{3}\right]$ or $\mathrm{K}\left[\mathrm{Pt}(\mathrm{Meteug}) \mathrm{Cl}_{3}\right]$ with 8 -hydroxyquinolines (HOquiN) in suitable solvents (see Experimental) gave complexes $[\mathrm{PtCl}(\mathrm{Saf})(\mathrm{OquiN})](\mathbf{1}, \mathbf{2}, \mathbf{3})$ and $[\mathrm{PtCl}(\mathrm{Meteug})(\mathrm{OquiN})](\mathbf{4}, 5)$. Complexes 1-5 (Fig. 2) easily precipitate from reaction mixture because they are neutral and have large hydrophobic residues.

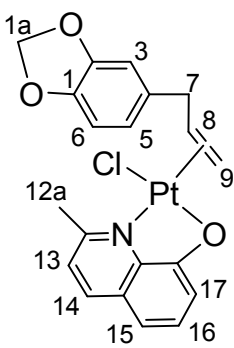

(1)

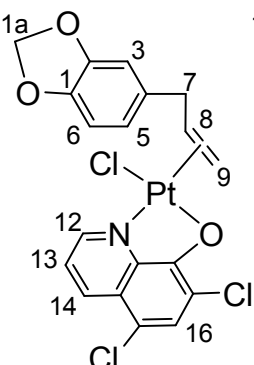

(2)

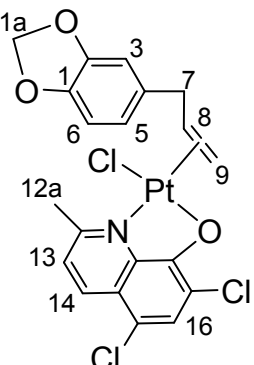

(3)

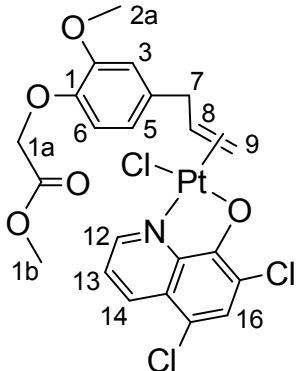

(4)

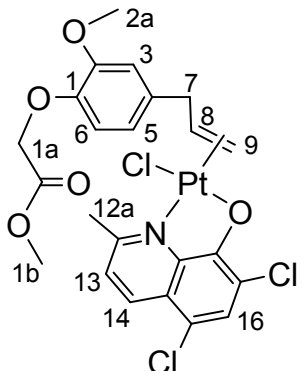

(5) 
Figure 2. Structure of complexes 1-5 (the numeration on these structuresis specifically used for NMR analysis).

In IR spectra of 1-5 (see Experimental) the absence of a band at about $1630 \mathrm{~cm}^{-1}$ from the $\mathrm{C}=\mathrm{C}$ double bond of allyl group in non-coordinated aryl olefin indicates that the allyl group coordinates in an $\eta^{2}$-manner. The absence of a strong absorption band at about $3400 \mathrm{~cm}^{-1}$ from the $\mathrm{OH}$ group of 8-hydroxyquinolines suggests that this $\mathrm{OH}$ group was deprotonated.

In the negative mode ESI MS of 3,4 and 5 there are pseudomolecular $[\mathrm{M}+\mathrm{Cl}]^{-}$peaks with relative intensity of 100, 40 and 100\%, respectively. In the positive mode ESI MS all of their pseudomolecular $[\mathrm{M}-\mathrm{Cl}]^{+}$peaks have relative intensity of $100 \%$, while their $[\mathrm{M}+\mathrm{H}]^{+}$peaks have relative intensity of 15,50 and $30 \%$, respectively. These are explained with the following reaction scheme.

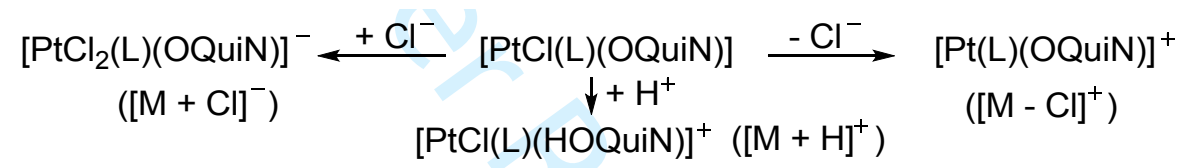

The assignment of the ${ }^{1} \mathrm{H}$ NMR signals of examined complexes is based on their chemical shift, spin-spin splitting patterns and intensities. For the ambiguous cases the NOESY spectra were also used. For example, the singlet of $\mathrm{H} 2 \mathrm{a}$ at $3.69 \mathrm{ppm}$ in complex $\mathbf{4}$ is distinguished from the near singlet of H1b_at 3.77 ppm by the fact that H2a has a NOESY cross peak with $\mathrm{H} 3$ while $\mathrm{H} 1 \mathrm{~b}$ has not. The ${ }^{1} \mathrm{H}$ NMR signals of 1-3 are listed in Table 2, and those of $\mathbf{4}$ and $\mathbf{5}$ are in Table 3.

The evidences for the coordination of the allyl group with Pt(II) in an $\eta^{2}$-manner are: i) The resonances of ethylenic protons (H8, H9cis, H9trans) shift upfield in comparison to those of non-coordinated allyl group and are accompanied by two ${ }^{195} \mathrm{Pt}$ satellites with the distance between them, ${ }^{2} J_{\mathrm{PtH}}, 70 \mathrm{~Hz}$ (Tables 2 and 3); ii) Two protons $\mathrm{H} 7$ give rise to two separate signals (denoted H7a and H7b, usually in form a doublet of doublets, Fig. 3) while for noncoordinated allyl group they give only one doublet. This is expected since upon coordination to $\mathrm{Pt}(\mathrm{II})$, atom $\mathrm{C} 8$ becomes a chiral center and thus $\mathrm{H} 7 \mathrm{a}$ and $\mathrm{H} 7 \mathrm{~b}$ become diastereotopic.

In ${ }^{1} \mathrm{H}$ NMR of 1-5, except the signal of moist water in $\mathrm{CDCl}_{3}$ at $1.5 \mathrm{ppm}$ there is not any HO-signal of used 8-hydroxyquinolines. The resonances of 2-methyl group of the coordinated 2-methylquinolin-8-olates (H12a of 1, 3 and 5 at 3.17, 3.19 and 3.17 ppm) largely shift 
downfield in comparison to those of non-coordinated 2-methyl-8-hydroxyquinolines (at about $2.5 \mathrm{ppm})$. These support the coordination of the chelating quinolin-8-olates with $\mathrm{Pt}(\mathrm{II})$.

In NOESY spectrum of 3 (Fig. 3) there is not any cross peak between all protons of the chelating OquiN including methyl proton $\mathrm{H} 12 \mathrm{a}$ and the protons of methyl eugenoxyacetate. In the spectrum there are cross peak $a$ shown the proximity of H12a to H13 and cross peak $b$ shown the proximity of $\mathrm{H} 9$ cis and H9trans to H8. That is to say, the donor $\mathrm{N}$ of the chelating OquiN and the ethylenic double bond $\mathrm{C} 8=\mathrm{C} 9$ are not at cis-positions but at trans-positions to one another in the coordination sphere of $\mathrm{Pt}(\mathrm{II})$.

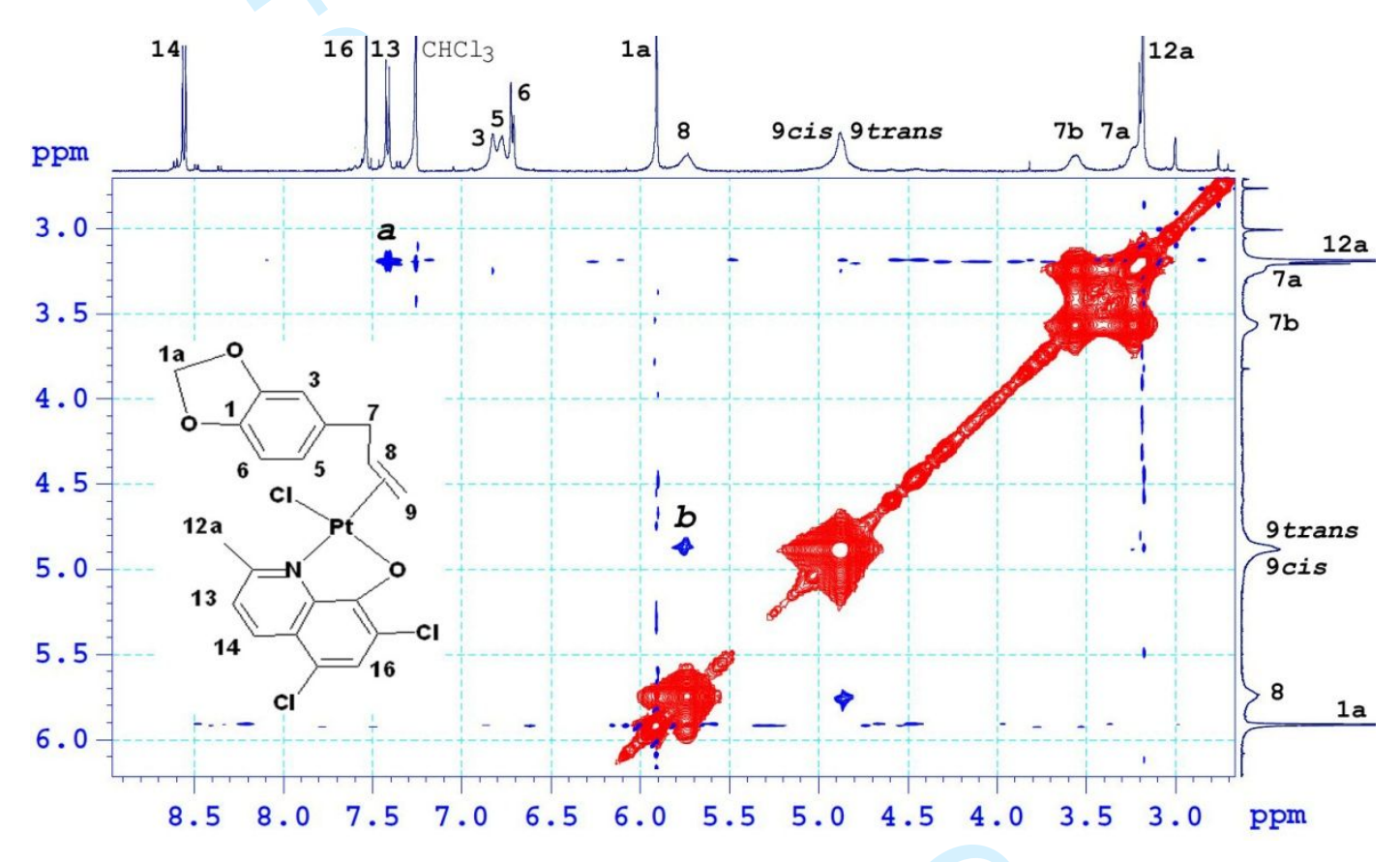

Figure 3. NOESY spectrum of complex 3 .

\subsection{Synthesis and structure of complexes 6-12}

Interaction of $\left[\mathrm{Pt}_{2} \mathrm{Cl}_{2}(\mathrm{Saf}-1 \mathrm{H})_{2}\right]$ or $\left[\mathrm{Pt}_{2} \mathrm{Cl}_{2}(\text { Eteug-1H })_{2}\right]$ with 8-hydroxyquinolines (HOquiN) in acetone/ethanol/chloroform (see Experimental) afforded complexes [Pt(Saf-1H)(OquiN)] (6, 7, 8, Fig. 4) and [Pt(Eteug-1H)(OquiN)] (9-12, Fig. 5). 


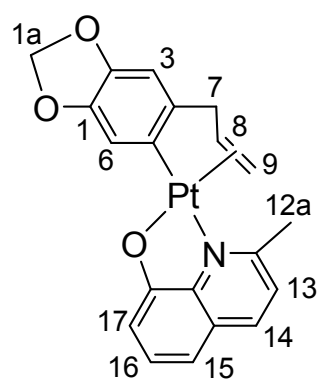

(6)

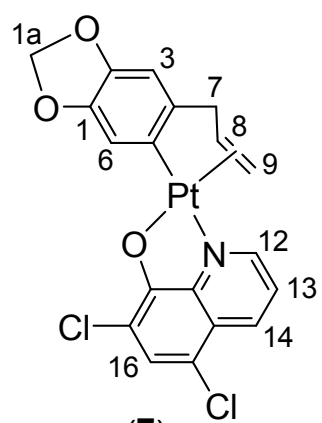

(7)

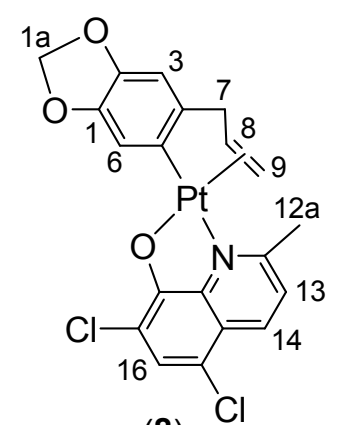

(8)

Figure 4. Structure of complexes 6-8.

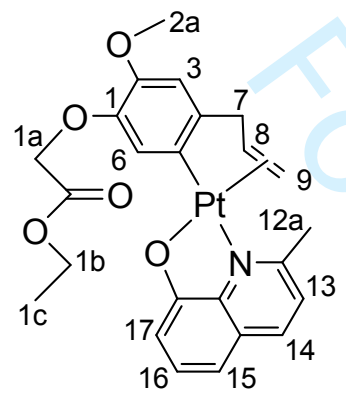

(9)

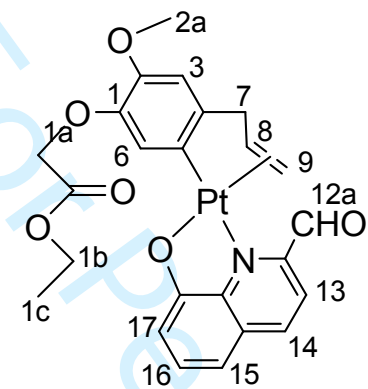

(10)

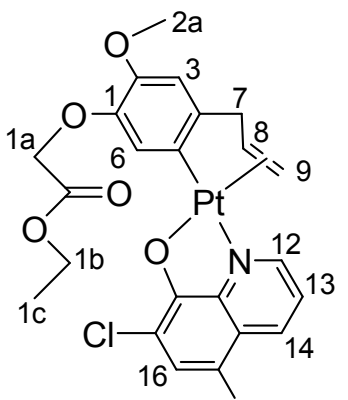

(11)

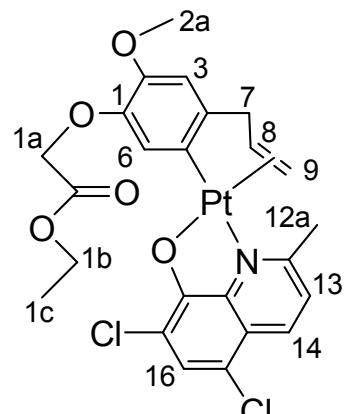

(12)

Figure 5. Structure of complexes 9-12 (the numeration on these structures is specifically used for NMR analysis).

The IR spectra of 6-8_and 9-12 are similar to those of 1-3_and 4-5 respectively (see 3.1). Although there is no $\mathrm{Cl}^{-}$ligandin complexes_8, 10_and_12, in their negative mode ESI MS there are pseudomolecular $[\mathrm{M}+\mathrm{Cl}]^{-}$peaks with relative intensity of $100 \%$. In the positive mode ESI MS of_8, 10_and_12 pseudomolecular $[\mathrm{M}+\mathrm{H}]^{+}$peaks have relative intensity of 15,50 and 30\%, respectively. These are associated with the following reaction scheme.

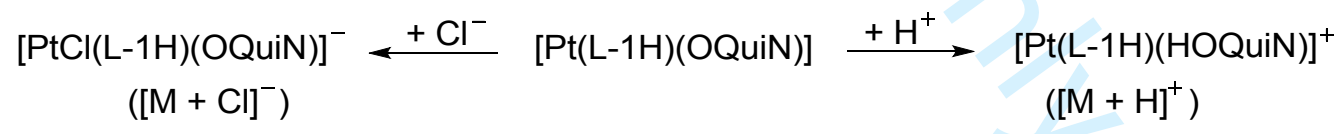

The evidences for the coordination of the allyl group with Pt(II) in 6-12 are similar to those for 1-5 (Tables 2 and 3). For complexes 6-12, the phenyl group gives rise to two signals: $\mathrm{H} 3$ and H6. The H6-singlet is distinguished from the $\mathrm{H} 3$-singlet by ${ }^{195} \mathrm{Pt}$ satellites with a ${ }^{3} J_{\mathrm{PtH}}$ value of $35 \mathrm{~Hz}$ (Table 2 and 3, Fig. 6). This value is comparable in magnitude to ${ }^{3} J_{\mathrm{PtH}}$ in analogous platinum(II) complexes $[11,12,14]$ and_twice smaller than ${ }^{2} J_{\mathrm{PtH}}(70-75 \mathrm{~Hz})$, indicating that the phenyl group is bound with $\mathrm{Pt}(\mathrm{II})$ by $\sigma-(\mathrm{C} 5-\mathrm{Pt})$-bond as shown in Fig. 4 and 5. The ${ }^{1} \mathrm{H}$ NMR signals of 6-8 are listed in Table 2, and those of 9-12 are in Table 3. The NOESY spectrum of $\mathbf{8}$ is presented in Fig. 6.

In Fig. 6 the cross peaks $a$ and $b$ shown the spatial proximity of H12a to H9cis and to H8. This indicates that the donor $\mathrm{N}$ of the chelating 'OquiN' is at cis-positions to the ethylenic 
double bond $\mathrm{C} 8=\mathrm{C} 9$ and the latter is almost perpendicular to the coordination plane of $\mathrm{Pt}(\mathrm{II})$, i.e. perpendicular to the plane of the chelating OquiN. Besides, there are the cross peaks which shown the proximity of $\mathrm{H} 12 \mathrm{a}$ to $\mathrm{H} 13$ (the cross peak c), H7a and H7b to H3 (the cross peaks $d$ and $e$ ), and so forth.

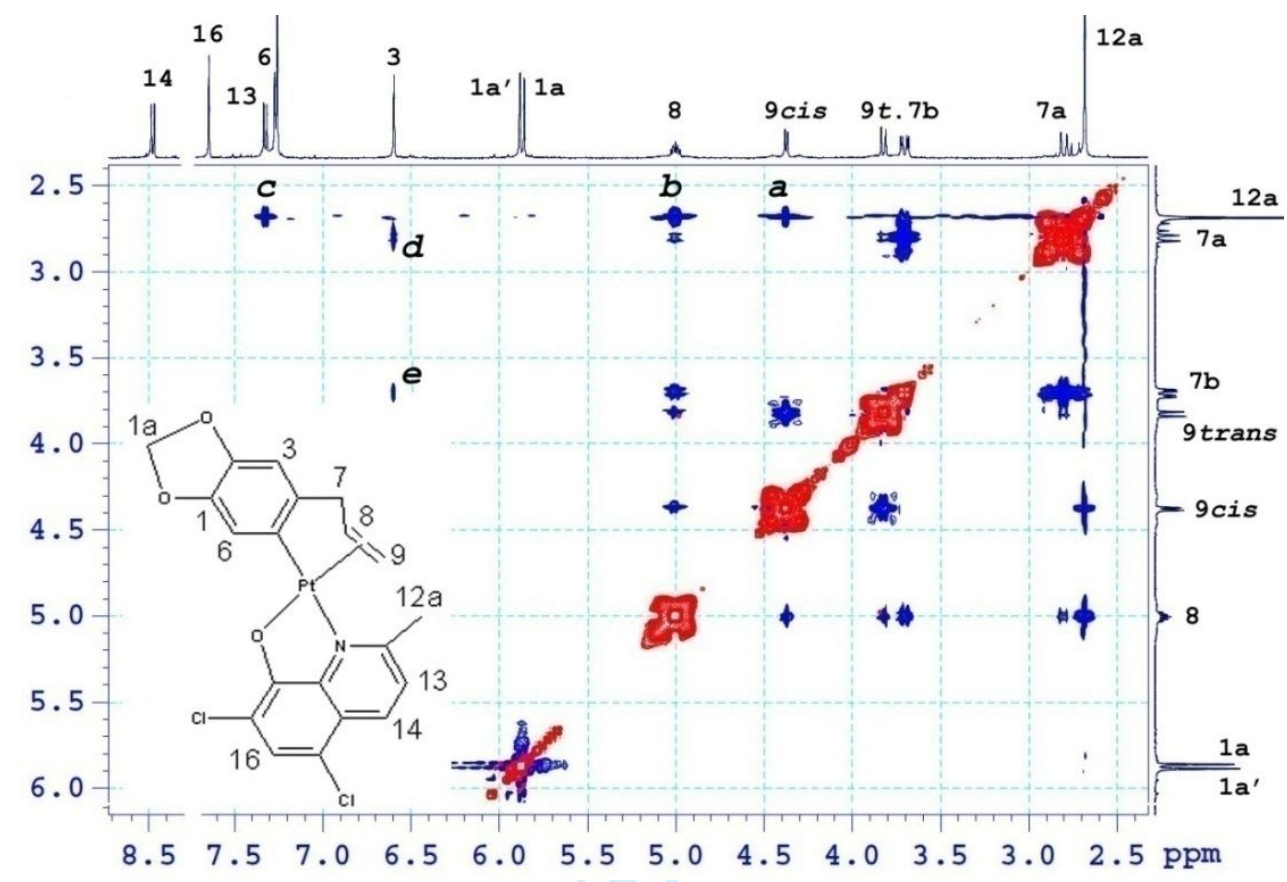

Figure 6. NOESY spectrum of complex 8 .

It is known that the interaction of $\left[\mathrm{Pt}_{2}\left(\mathrm{C}_{2} \mathrm{H}_{4}\right)_{2} \mathrm{Cl}_{4}\right]$ and amines (Am) gives trans$\left[\mathrm{Pt}\left(\mathrm{C}_{2} \mathrm{H}_{4}\right)(\mathrm{Am}) \mathrm{Cl}_{2}\right]$, as ethylene has a large trans effect. The formation of 1-5 obeys the transeffect (i.e. donor $\mathrm{N}$ and ethylenic double bond are at trans-positions to one another), whilst in the interaction of $\left[\mathrm{Pt}_{2} \mathrm{Cl}_{2}(\mathrm{Saf}-1 \mathrm{H})_{2}\right]$ or $\left[\mathrm{Pt}_{2} \mathrm{Cl}_{2}(\text { Eteug- } 1 \mathrm{H})_{2}\right]$ with 8-hydroxyquinolines (HOquiN) donor $\mathrm{N}$ was not introduced trans to the ethylenic double bond of the allyl group but were trans to C5 of the phenyl group to form complexes 6-12 (Fig. 4 and 5). We suggest that this selectivity is controlled by steric effects rather than by the trans effect.

It is interesting to note some anomalies in the ${ }^{1} \mathrm{H}$ NMR spectra of the examined complexes (Tables 2 and 3). First, for 6-12, the chemical shift of H7a (2.7-2.9 ppm) is largely decreased in comparision with those of $\mathrm{H} 7 \mathrm{~b}$ (3.6-3.8 ppm). Second, for 1-3, two methylene protons H1a and H1a' are equivalent, while_for 6-8 they are non equivalent (Table 2). Third, the chemical shift of 2-metyl protons of OquiN ligand (H12a) in 6, 8, 9 and 12 (2.67-2.69 ppm) is much smaller than those in $\mathbf{1}, \mathbf{3}$ and $\mathbf{5}$ (3.17-3.19 ppm). 
The first and the second observations are related to the formation_of a non-coplanar platinacycle from the allyl, phenyl groups and the Pt(II) as seen from Fig. 4, 5 and 7. For 6-12 this cyclization causes a steric compressed $\mathrm{Pt}(\mathrm{C} 7-\mathrm{C} 8-\mathrm{C} 9)$-system increasing the spatial difference between $\mathrm{H} 7 \mathrm{a}$ and $7 \mathrm{~b}$. For 6-8 this cyclization causes some deformation of the fivemembered 1,3-dioxole ring making its two methylene protons become non-equivalent. In addition, it is surprising that for 9-12 two methylene protons H1a and H1a' as well as H1b and H1b' are non-equivalent (Table 3). It is possible that $-\mathrm{OCH}_{2} \mathrm{COOCH}_{2} \mathrm{CH}_{3}$ as bulky group hinders the rotations around its single $\mathrm{C}-\mathrm{O}$ bonds. The third one involves the spatial proximity of $\mathrm{H} 12 \mathrm{a}$ to the double bond $\mathrm{C} 8=\mathrm{C} 9$ in $\mathbf{6 , 8}, \mathbf{9}$ and $\mathbf{1 2}$. As a result, the methyl protons $\mathrm{H} 12 \mathrm{a}$ fall into the shielded region of the double bond $\mathrm{C} 8=\mathrm{C} 9$ and therefore they resonate at relative high field (see also Fig. 7).

\subsection{Crystal structure of complex 9}

The molecular structure of complex 9 determined by single-crystal X-ray diffractionis shown in Fig. 7 (a different numbering scheme is used than in Fig. 5). The $\mathrm{Pt}(\mathrm{II})$ atom is surrounded by an aromatic $\mathrm{C}$ atom and the $\mathrm{C}=\mathrm{C}$ double bond of the ethyl eugenoxyacetate ligand, and by the $\mathrm{N}$ and $\mathrm{O}$ atoms of the 8-oxyquinoline, resulting in a slightly distorted square-planar coordination environment and the the $\mathrm{N}$ and $\mathrm{O}$ atoms are in transpositions (the trans angle C14-Pt1-N2 is $\left.169.80(9)^{\circ}\right)$. The best planes through both aromatic rings make an angle of $41.41(10)^{\circ}$. In general, these confirm the solution structure of the examined complexes.

It is noted that for platinum(II) complexes containing natural aryl olefin and monodentate amine, the single-crystal X-ray diffraction data were reported in several works $[11,13,15]$. For platinum(II) complexes containing natural aryl olefin and didentate amine, the positions of $\mathrm{N}$ and $\mathrm{O}$ donors in platinum(II) coordination sphere was usually determined by NMR data $[10$, 13], in the present paper these position were determined by both NMR and the single-crystal X$\underline{\text { ray diffraction investigations. }}$

\subsection{Biological activities of complexes $7,9,11,12$}

Four complexes 7, 9, 11 and 12 were tested for cell in vitro cytotoxicity on human cancer cells and the $\mathrm{IC}_{50}$ values are listed in Table 4. [Pt(Saf-1H)(5,7-Cl $\mathrm{C}_{2}-8$-O-quinoline)] (7) exhibits high activities on four cell lines Human epidermic carcinoma (KB), Hepatocellular carcinoma (Hep-G2), Human lung carcinoma (Lu), and Human breast carcinoma (MCF-7)

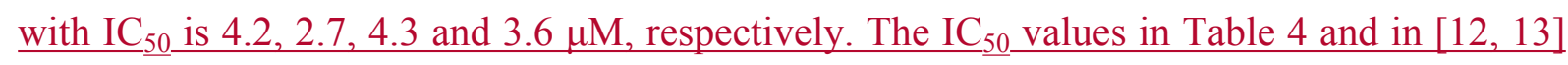


shown that the complexes containing safrole or chelating safrole usually give higher cytotoxicity than the complexes containing ethyl eugenoxyacetate.-
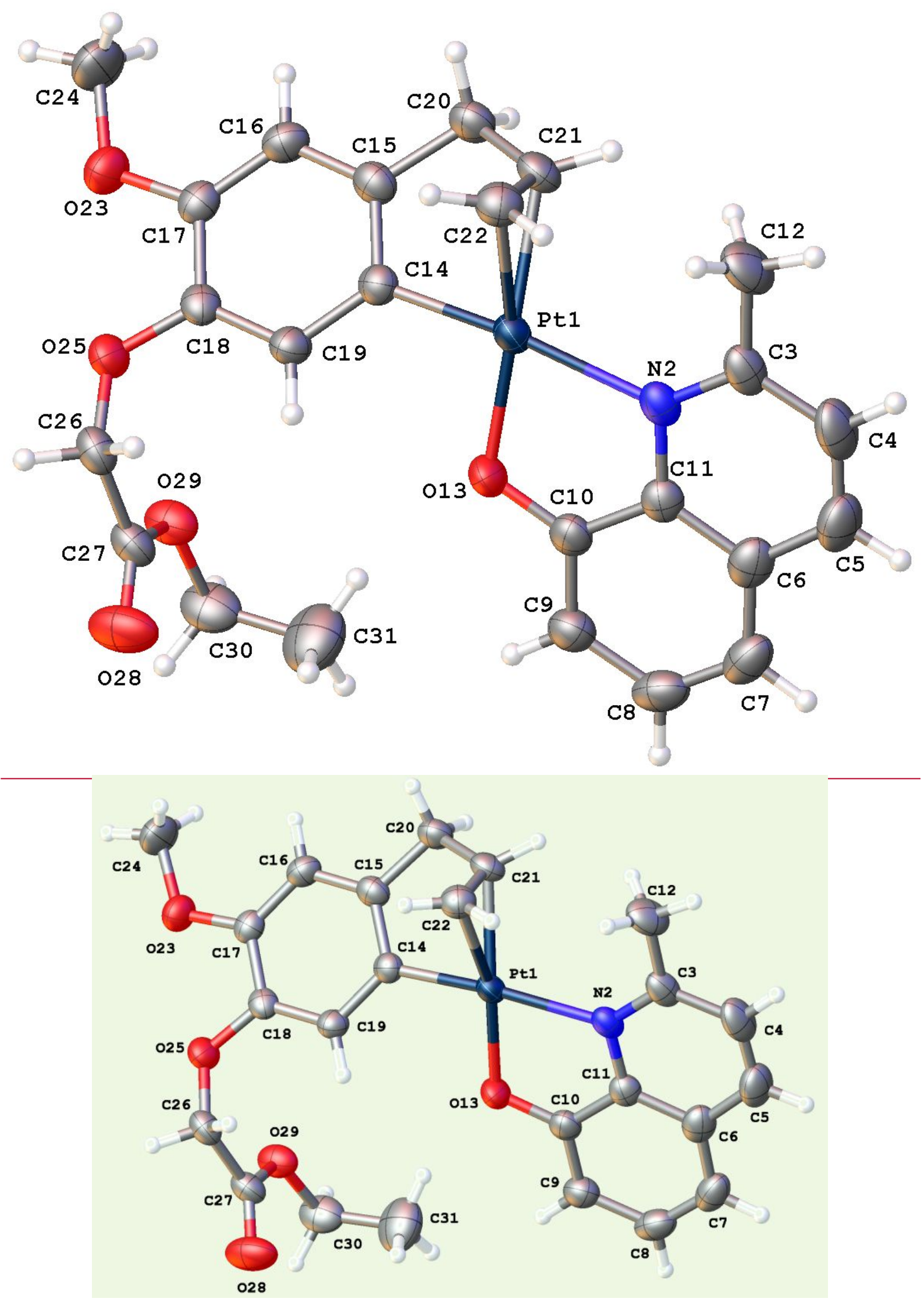
Figure 7. Molecular structure of 9, with atom labelling scheme and displacement ellipsoids drawn at the $50 \%$ probability level.

Table 4. The cell in vitro cytotoxicity of examined compounds, $\mathrm{IC}_{50}(\mu \mathrm{M})$.

\begin{tabular}{|c|c|c|c|c|}
\hline Cell line & KB & Hep-G2 & $\mathrm{Lu}$ & MCF-7 \\
\hline$\left[\mathrm{Pt}(\mathrm{Saf}-1 \mathrm{H})\left(5,7-\mathrm{Cl}_{2}-8-\mathrm{O}\right.\right.$-quinoline $\left.)\right](7)$ & 4.2 & 2.7 & 4.3 & 3.6 \\
\hline [Pt (Eteug-1H)(2-Me-8-O-quinoline)] (9) & $>212$ & $>212$ & - & - \\
\hline$\left[\mathrm{Pt}\left(\right.\right.$ Eteug-1H)(5,7-Cl${ }_{2}-8-\mathrm{O}$-quinoline $\left.)\right](\mathbf{1 1})$ & 121.3 & 25.3 & - & - \\
\hline$\left[\mathrm{Pt}\left(\right.\right.$ Eteug-1H)(5,7-Cl ${ }_{2}-2-\mathrm{Me}-8-\mathrm{O}$-quinoline $\left.)\right](\mathbf{1 2})$ & 16.4 & 74.8 & 66.5 & 22.0 \\
\hline 8-HO-quinoline & 37.9 & 43.5 & 62.9 & 40.4 \\
\hline
\end{tabular}

\section{Conclusions}

In this work, twelve new organoplatinum(II) complexes were synthesized from $\mathrm{K}\left[\mathrm{Pt}(\mathrm{Saf}) \mathrm{Cl}_{3}\right]$ (Saf: Safrole), K[Pt(Meteug)Cl $\left.{ }_{3}\right]$ (Meteug: Methyl eugenoxyacetate), $\left[\mathrm{Pt}_{2} \mathrm{Cl}_{2}(\mathrm{Saf}-1 \mathrm{H})_{2}\right]$ (Saf$1 \mathrm{H}:$ Deprotonated safrole), $\quad\left[\mathrm{Pt}_{2} \mathrm{Cl}_{2}(\text { Eteug-1H })_{2}\right]$ (Eteug-1H: Deprotonated ethyl eugenoxyacetate) and three substituted 8-hydroxyquinolines (HOquiN). The obtained complexes are: [PtCl(Saf)(2-Me-8-OquiN) $]$ (1), $\left[\mathrm{PtCl}(\mathrm{Saf})\left(5,7-\mathrm{Cl}_{2}-8-\mathrm{OquiN}\right)\right] \quad$ (2), $\left[\mathrm{PtCl}(\mathrm{Saf})\left(5,7-\mathrm{Cl}_{2}-2-\mathrm{Me}-8-\mathrm{OquiN}\right)\right] \quad$ (3), [PtCl(Meteug) $\left.\left(5,7-\mathrm{Cl}_{2}-8-\mathrm{OquiN}\right)\right] \quad$ (4), [PtCl(Meteug)(5,7-Cl $-2-M e-8-O q u i N)] \quad$ (5), [Pt(Saf-1H)(2-Me-8-OquiN) $\quad$ (6), [Pt(Saf$\left.1 \mathrm{H})\left(5,7-\mathrm{Cl}_{2}-8-\mathrm{OquiN}\right)\right](7),\left[\mathrm{Pt}(\mathrm{Saf}-1 \mathrm{H})\left(5,7-\mathrm{Cl}_{2}-2-\mathrm{Me}-8-\mathrm{OquiN}\right)\right](8),[\mathrm{Pt}($ Eteug-1H)(2-Me-8OquiN)] (9), [Pt(Eteug-1H)(2-OHC-8-OquiN)] (10), [Pt(Eteug-1H)(5,7-Cl $2-8-$ OquiN)] (11), and $[\mathrm{Pt}($ Eteug-1H)(5,7-Cl $2-2-\mathrm{Me}-8-\mathrm{OquiN})]$ (12). These complexes were characterized by elemental analyses, IR, ${ }^{1} \mathrm{H}$ NMR, for several also by MS spectra and single crystal X-ray diffraction._The exhaustive study on these spectra shown that in 1-5 the donor $\mathrm{N}$ of the substituted quinolin-8-olates is in trans-position, and in 6-12 the donor $\mathrm{N}$ is in cis-position in comparison with the ethylenic double bond of the allyl group. Four complexes were tested for cell in vitro cytotoxicity on human cancer cells. Complex 7 exhibits high activities on human cancer cells $\mathrm{KB}$, Hep-G2, $\mathrm{Lu}$ and MCF-7 with $\mathrm{IC}_{50}$ values of 4.2, 2.7, 4.3 and $3.6 \mu \mathrm{M}$, respectively.

\section{Acknowledgements}


This research is funded by Vietnam Ministry of Education under grant number B 2017-SPH43. LVM thanks the Hercules Foundation for supporting the purchase of the diffractometer through project AKUL/09/0035.

Appendix A. Supplementary data

CCDC 1856503 contain the supplementary crystallographic data for complexes 9 . These data can be obtained free of charge via http://www.ccdc.cam.ac.uk/conts/retrieving.html, or from the Cambridge Crystallographic Data Centre, 12 Union Road, Cambridge CB2 1EZ, UK; fax: (+44) 1223-336-033; or e-mail: deposit@ccdc.cam.ac.uk.

\section{References}

[1] G. Pneumatikakis, Inorganica Chimica Acta, 93, 5-11 (1984).

[2] T.alal-A.K. Al-Allaf, M.ikdad T. Ayoub, L.tay J. Rashan, Journal of Inorganic Biochemistry, 38, 47-56 (1990).

[3] A. L. Konovalova, K. I. Yakovlev, A. I. Stetsenko, N. D. Rozhkova, G. K. Gerasimova, T. I. Ivanova, N. S. Kamaletdinov, and V. P. Sinditskii, Pharmaceutical Chemistry Journal, 28, 21-24 (1994).

[4] L. Fang, M. Feng, F. Chen, X. Liu, H. Shen, J. Zhao, S. Gou, Bioorganic \& Medicinal Chemistry. 24, 4611 (2016).

[5] M. Massoni, J. C. Tenorio Clavijo, L. Colina-Vegas, W. Villarreal, J. S. M. Dias, G. A. F. da Silva, M. Ionta, M. Soares, J. Ellena, A. C. Dorigueto, M. I. F. Barbosa, A. A. Batista, Polyhedron, 129, 214-221 (2017).

[6] C. M. Santos, S. Cabrera, C. Ríos-Luci, J. M. Padrón, I. L. Solera, A. G. Quiroga, M. A. Medrano, C. Navarro-Ranninger and J. Alemán,_Dalton Trans., 42, 13343-13348 (2013).

[7] M. D. Živković, J. Kljun, T. Ilic-Tomic, A. Pavic, A. Veselinović, D. D. Manojlović, J. Nikodinovic-Runic and I. TurelInorg. Inorg.Chem. Front., 5, 39-53 (2018).

[8] T. T. Da, N. T. T. Minh, N. T. T. Chi, N. H. Dinh. Polyhedron, 26, 3271-3276 (2007).

[9] T. T. Da, Y. M. Kim, N. T. T. Chi, L. X. Chien, N. V. Minh, N. H. Dinh.

Organometallics, 27, 3611-3613 (2008).

[10] N. T. T. Chi, T. T. Da, N. V. Ha, N. H. Dinh, Journal of Coordination Chemistry, 70, 1008-1017 (2017).

[11] T. T. Da, Y. M. Kim, T. T. C. Mai, N. C. Cuong and N. H. Dinh. Journal of Coordination Chemistry, 63, 473-483 (2010).

[12] T. T. Da, L. X. Chien, N. T. T. Chi, L. T. H. Hai and N. H. Dinh. Journal of Coordination Chemistry, 65, 131-142 (2012). 
[13] T. T. Da, L. T. H. Hai, L. Van Meervelt, N. H. Dinh, Journal of Coordination Chemistry, 68, 3525-3536_(2015).

[14] T. T. Da, N. T. T. Chi, L. Van Meervelt, P. M. Kimpende and N. H. Dinh,Polyhedron, 85, 104-109 (2015).

[15] N. T. T. Chi, T. T. Da, K. Robeyns, L. Van Meervelt, T. T. C. Mai, N. D. Dat.and N. H. Dinh. Polyhedron, 151, 330-337 (2018).

16. V. Prachayasittikul, S. Prachayasittikul, S. Ruchirawat, V. Prachayasittiku, Drug Design, Development and Therapy, 7, 1157-1178(2013).

[17] C. M. Santos, S. Cabrera, C. Rios-Luci, J. M. Padron, I. L. Solera, A. G. Quiroga, M. A. Medrano, C. Navarro-Ranninger and J. Aleman,Dalton Trans., 42, 13343-13348 (2013).

[18] A. D. Ghelman, E. F. Karandasova, L. N. Essen, Izv. Sectora Platinum, Inst. Obsch. Nheorg. Khim. AN SSSR , 24, 68-72 (1949).

[19] P. Skehan, R. Storeng, D. Scudiero, A. Monks, J. McMahon, D. Vistica, JT. Warren, H. Bokesch, S. Kenney, MR. Boyd, J. National Cancer Institute, 82, 1107-1112 (1990).

[20] CrysAlis PRO. Agilent Technologies UK Ltd, Yarnton, Oxfordshire, England, (2012).

[21] O. V. Dolomanov, L. J. Bourhis, R. J. Gildea, J. A. K. Howard, and H. Puschmann, J. Appl. Cryst. 42(2009), 339-341_(2009).

[22] G. M. Sheldrick, Acta Crystallogr. A64, 112-122 (2008).

[23] G.M. Sheldrick, Acta Crystallogr. C71, 3-8 (2015). 
Table 1. Crystal data and refinement details for crystal structures of complex 9.

\begin{tabular}{|l|l||l|l|}
\hline Formula & $\mathrm{C}_{24} \mathrm{H}_{25} \mathrm{NO}_{5} \mathrm{Pt}$ & $V\left(\AA^{3}\right)$ & $1061.78(5)$ \\
\hline Formula weight & 602.54 & $Z$ & 2 \\
\hline Crystal size $(\mathrm{mm})$ & $0.25 \times 0.2 \times 0.2$ & $\rho_{\text {calc }}\left(\mathrm{g} / \mathrm{cm}^{3}\right)$ & 1.885 \\
\hline Radiation & $\mathrm{Mo} \mathrm{K} \alpha(\lambda=0.71073)$ & $\mu\left(\mathrm{mm}^{-1}\right)$ & 6.644 \\
\hline Temperature $(\mathrm{K})$ & $293.4(6)$ & $F(000)$ & 588.0 \\
\hline Crystal system & triclinic & $2 \theta$ value $\left(^{\circ}\right)$ & $5.222-52.763$ \\
\hline Space group & $P-1$ & Reflections collected & 21675 \\
\hline$a(\AA)$ & $9.3398(3)$ & Independent reflections & 4316 \\
\hline$b(\AA)$ & $10.3892(3)$ & $R_{\text {int }}$ & 0.0376 \\
\hline$c(\AA)$ & $11.4404(3)$ & Data/restraints $/$ parameters & $4316 / 0 / 283$ \\
\hline$\alpha\left(^{\circ}\right)$ & $92.004(2)$ & $R\left[F^{2}>2 \sigma\left(F^{2}\right)\right], w R\left(F^{2}\right)$ & $0.0163,0.0365$ \\
\hline$\beta\left(^{\circ}\right)$ & $104.825(2)$ & Goodness-of-fit on $\mathrm{F}^{2}$ & 1.074 \\
\hline$\gamma\left(^{\circ}\right)$ & $97.310(2)$ & Largest diff. peak, hole $\left(\mathrm{e} \AA^{-3}\right)$ & $0.43,-0.58$ \\
\hline
\end{tabular}


Table 2. ${ }^{1} \mathrm{H}$ NMR signals of complexes 1-3 and 6-8, $\delta(\mathrm{ppm}), J(\mathrm{~Hz})$.

\begin{tabular}{|c|c|c|c|c|c|c|}
\hline & $\mathbf{1} / \mathrm{CDCl} 3$ & $2 / \mathrm{CDCl} 3$ & 3/CDCl3 & 6/CDCl3 & $7 / \mathrm{CDCl} 3$ & $\mathbf{8} / \mathrm{CDCl3}$ \\
\hline H1a & $5.91 \mathrm{~s}$ & $5.91 \mathrm{~s}$ & $5.91 \mathrm{~s}$ & $5.84 \mathrm{~d} ;{ }^{2} J 1$ & $5.85 \mathrm{~d} ;{ }^{2} J 1$ & $5.86 \mathrm{~d} ;{ }^{2} J 1$ \\
\hline H1a' & $5.91 \mathrm{~s}$ & $5.91 \mathrm{~s}$ & $5.91 \mathrm{~s}$ & $5.87 \mathrm{~d} ;{ }^{2} J 1$ & $5.88 \mathrm{~d} ;{ }^{2} J 1$ & $5.89 \mathrm{~d} ;{ }^{2} J 1$ \\
\hline $\mathrm{H} 3$ & $6.84 \mathrm{~s}$ & $6.83 \mathrm{~d} ;{ }^{4} J 1.5$ & $6.83 \mathrm{~s}$ & $6.61 \mathrm{~s}$ & $6.61 \mathrm{~s}$ & $6.60 \mathrm{~s}$ \\
\hline H5 & $6.79 \mathrm{~d} ;{ }^{3} J 7.5$ & $\begin{array}{c}6.78 \mathrm{dd} ; \\
{ }^{3} J 8 ;{ }^{4} J 1.5\end{array}$ & $6.78 \mathrm{~d} ;{ }^{3} J 7.5$ & - & - & - \\
\hline H6 & $6.72 \mathrm{~d} ;{ }^{3} J 7.5$ & $6.71 \mathrm{~d} ;{ }^{3} J 8$ & $6.72 \mathrm{~d} ;{ }^{3} J 7.5$ & $7.23 \mathrm{~s}$ & $\begin{array}{l}7.18 \mathrm{~s} ; \\
{ }^{3} J_{\mathrm{PtH}} 35\end{array}$ & $\begin{array}{c}7.27 \mathrm{~s} ; \\
{ }^{3} J_{\mathrm{PtH}} \mathrm{OV} .\end{array}$ \\
\hline $\mathrm{H} 7 \mathrm{a}$ & 3.21 br. & $\begin{array}{c}3.33 \mathrm{dd} ; \\
{ }^{2} J 15 ;{ }^{3} J 8\end{array}$ & $\begin{array}{c}3.22 \mathrm{dd} ; \\
{ }^{2} J 15 ;{ }^{3} J 7\end{array}$ & $\begin{array}{c}2.82 \mathrm{~d} ;{ }^{2} J 17 \\
{ }^{3} J_{\mathrm{PtH}} 95\end{array}$ & $\begin{array}{c}2.85 \mathrm{~d} ;{ }^{2} J 17 ; \\
{ }^{3} J_{\mathrm{PtH}} 95\end{array}$ & $\begin{array}{c}2.81 \mathrm{~d} ;{ }^{2} J 17 ; \\
{ }^{3} J_{\mathrm{PtH}} 95\end{array}$ \\
\hline $\mathrm{H} 7 \mathrm{~b}$ & $3.57 \mathrm{br}$. & $\begin{array}{c}3.63 \mathrm{dd} ; \\
{ }^{2} J 15 ;{ }^{3} J 6\end{array}$ & $\begin{array}{c}3.56 \mathrm{~d} ; \\
2 J 15\end{array}$ & $\begin{array}{c}3.74 \mathrm{dd} ; \\
{ }^{2} J 17 ;{ }^{3} J 6.5\end{array}$ & $\begin{array}{c}3.69 \mathrm{dd} ; \\
{ }^{2} J 17 ;{ }^{3} J 6\end{array}$ & $\begin{array}{c}3.71 \mathrm{dd} ; \\
{ }^{2} J 17 ;{ }^{3} J 6\end{array}$ \\
\hline H8 & $\begin{array}{l}5.65 \mathrm{~m} ; \\
{ }^{2} J_{\mathrm{PtH}} 70\end{array}$ & $\begin{array}{l}5.75 \mathrm{~m} \\
{ }^{2} J_{\mathrm{PtH}} 70\end{array}$ & $\begin{array}{l}5.73 \mathrm{~m} ; \\
{ }^{2} J_{\mathrm{PtH}} 70\end{array}$ & $\begin{array}{l}4.98 \mathrm{~m} ; \\
{ }^{2} J_{\mathrm{PtH}} 75\end{array}$ & $\begin{array}{l}4.78 \mathrm{~m} ; \\
{ }^{2} J_{\mathrm{PtH}} 75\end{array}$ & $\begin{array}{l}5.01 \mathrm{~m} ; \\
{ }^{2} J_{\mathrm{PtH}} 75\end{array}$ \\
\hline H9cis & $\begin{array}{c}4.78 \mathrm{~d} ;{ }^{3} J 7 \\
{ }^{2} J_{\mathrm{PtH}} 70\end{array}$ & $\begin{array}{c}4.92 \mathrm{~d} ;{ }^{3} J 8 \\
{ }^{2} J_{\mathrm{PtH}} 70\end{array}$ & $\begin{array}{c}4.87 \mathrm{~d} ;{ }^{3} J 8 \\
{ }^{2} J_{\mathrm{PtH}} 70\end{array}$ & $\begin{array}{c}4.35 \mathrm{~d} ;{ }^{3} J 7.5 \\
{ }^{2} J_{\mathrm{PtH}} 75\end{array}$ & $\begin{array}{c}4.07 \mathrm{~d} ;{ }^{3} J 8 \\
{ }^{2} J_{\mathrm{PtH}} 75\end{array}$ & $\begin{array}{c}4.38 \mathrm{~d} ;{ }^{3} J 8 \\
{ }^{2} J_{\mathrm{PtH}} 75\end{array}$ \\
\hline H9trans & $\begin{array}{c}4.75 \mathrm{~d} ;{ }^{3} J 14 \\
{ }^{2} J_{\mathrm{PtH}} 70\end{array}$ & $\begin{array}{c}4.88 \mathrm{~d} ;{ }^{3} J 15 \\
{ }^{2} J_{\mathrm{PtH}} 70\end{array}$ & $\begin{array}{c}4.86 \mathrm{~d} ;{ }^{3} J 13 ; \\
{ }^{2} J_{\mathrm{PtH}} 70\end{array}$ & $\begin{array}{c}3.72 \mathrm{~d} ;{ }^{3} J 13 \\
{ }^{2} J_{\mathrm{PtH}} 75\end{array}$ & $\begin{array}{c}3.93 \mathrm{~d} ;{ }^{3} J 13 \\
{ }^{2} J_{\mathrm{PtH}} 75\end{array}$ & $\begin{array}{c}3.83 \mathrm{~d} ;{ }^{3} J 13 ; \\
{ }^{2} J_{\mathrm{PtH}} 75\end{array}$ \\
\hline $\mathrm{H} 12$ & - & $\begin{array}{c}9.16 \mathrm{dd} ; \\
{ }^{3} J 5.5 ;{ }^{4} J 1.5\end{array}$ & 8 & - & $8.15 \mathrm{~d} ;{ }^{3} J 5$ & - \\
\hline $\mathrm{H} 12 \mathrm{a}$ & $3.17 \mathrm{~s}$ & - & $3.19 \mathrm{~s}$ & $2.67 \mathrm{~s}$ & - & $2.69 \mathrm{~s}$ \\
\hline H13 & $7.31 \mathrm{~d} ;{ }^{3} J 8.5$ & $\begin{array}{c}7.68 \mathrm{dd} ; \\
{ }^{3} J 8.5 ;{ }^{3} J 5.5\end{array}$ & $\begin{array}{l}7.42 \mathrm{~d} ; \\
{ }^{3} J 8.5\end{array}$ & $7.24 \mathrm{~d} ;{ }^{3} J 8.5$ & $\begin{array}{c}7.56 \mathrm{dd} ;{ }^{3} J \\
8.5 ;{ }^{3} J 5\end{array}$ & $7.33 \mathrm{~d} ;{ }^{3} J 8.5$ \\
\hline H14 & $8.22 \mathrm{~d} ;{ }^{3} J 8.5$ & $\begin{array}{l}8.75 \mathrm{dd} ;{ }^{3} J \\
8.5 ;{ }^{4} J 1.5\end{array}$ & $\begin{array}{l}8.56 \mathrm{~d} ; \\
{ }^{3} J 8.5 ;\end{array}$ & $8.15 \mathrm{~d} ;{ }^{3} J 8.5$ & $8.66 \mathrm{~d} ;{ }^{3} J 8.5$ & $8.49 \mathrm{~d} ;{ }^{3} J 8.5$ \\
\hline H15 & $6.96 \mathrm{~d} ;{ }^{3} J 7.5$ & - & - & $7.20 \mathrm{~d} ;{ }^{3} J 8$ & - & - \\
\hline H16 & $7.33 \mathrm{t} ;{ }^{3} J 7.5$ & $7.64 \mathrm{~s}$ & $7.54 \mathrm{~s}$ & $7.46 \mathrm{t} ;{ }^{3} J 8$ & $7.74 \mathrm{~s}$ & $7.65 \mathrm{~s}$ \\
\hline H17 & $6.96 \mathrm{~d} ;{ }^{3} J 7.5$ & - & - & $7.00 \mathrm{~d} ;{ }^{3} J 8$ & 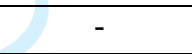 & - \\
\hline
\end{tabular}


Table 3. ${ }^{1} \mathrm{H}$ NMR signals of complexes 4, 5 and 9-12, $\delta(\mathrm{ppm}), J(\mathrm{~Hz})$.

\begin{tabular}{|c|c|c|c|c|c|c|}
\hline & $4 / \mathrm{CDCl}_{3}$ & $\mathbf{5} / \mathrm{CDCl}_{3}$ & $\mathbf{9} / \mathrm{Me}_{2} \mathrm{CO}$ & $\mathbf{1 0} / \mathrm{CDCl}_{3}$ & 11/ $\mathrm{Me}_{2} \mathrm{CO}$ & $\mathbf{1 2} / \mathrm{CDCl}_{3}$ \\
\hline H1a & $4.63 \mathrm{~s}$ & $4.63 \mathrm{~s}$ & $\begin{array}{l}4.57 \mathrm{~d} ;{ }^{2} J 16 \\
4.58 \mathrm{~d} ;{ }^{2} J 16\end{array}$ & $\begin{array}{l}4.76 \mathrm{~s} \\
4.76 \mathrm{~s}\end{array}$ & $\begin{array}{l}4.66 \mathrm{~s} \\
4.66 \mathrm{~s}\end{array}$ & $\begin{array}{l}4.78 \mathrm{~d} ;{ }^{2} J 16 \\
4.79 \mathrm{~d} ;{ }^{2} J 16\end{array}$ \\
\hline H1b & $3.77 \mathrm{~s}$ & $3.77 \mathrm{~s}$ & $\begin{array}{l}4.18 \mathrm{q} ;{ }^{3} J 7 \\
4.19 \mathrm{q} ;{ }^{3} J 7\end{array}$ & $\begin{array}{l}4.31 \mathrm{q} ;{ }^{3} J 7 \\
4.32 \mathrm{q} ;{ }^{3} J 7\end{array}$ & $\begin{array}{l}4.24 \mathrm{q} ;{ }^{3} J 7 \\
4.26 \mathrm{q} ;{ }^{3} J 7\end{array}$ & $\begin{array}{l}4.28 \mathrm{q} ;{ }^{3} J 7 \\
4.29 \mathrm{q} ;{ }^{3} J 7\end{array}$ \\
\hline $\mathrm{H} 1 \mathrm{c}$ & - & - & $1.23 \mathrm{t} ;{ }^{3} \mathrm{~J} 7$ & $1.33 \mathrm{t} ;{ }^{3} \mathrm{~J} 7$ & $1.28 \mathrm{t} ;{ }^{3} \mathrm{~J} 7$ & $1.31 \mathrm{t} ;{ }^{3} J 7$ \\
\hline $\mathrm{H} 2 \mathrm{a}$ & $3.69 \mathrm{~s}$ & $3.66 \mathrm{~s}$ & $3.69 \mathrm{~s}$ & $3.84 \mathrm{~s}$ & $3.76 \mathrm{~s}$ & $3.83 \mathrm{~s}$ \\
\hline $\mathrm{H} 3$ & $6.90 \mathrm{~s}$ & $6.89 \mathrm{~s}$ & $6.67 \mathrm{~s}$ & $6.70 \mathrm{~s}$ & $6.74 \mathrm{~s}$ & $6.66 \mathrm{~s}$ \\
\hline H5 & $6.86 \mathrm{~d} ;{ }^{3} J 8$ & $6.86 \mathrm{~d} ;{ }^{3} J 8$ & - & - & - & - \\
\hline H6 & $6.75 \mathrm{~d} ;{ }^{3} J 8$ & $6.75 \mathrm{~d} ;{ }^{3} J 8$ & $\begin{array}{c}7.13 \mathrm{~s} \\
{ }^{3} J_{\mathrm{PtH}} 35\end{array}$ & $\begin{array}{l}7.20 \mathrm{~s} ; \\
{ }^{3} J_{\mathrm{PtH}} 35\end{array}$ & $\begin{array}{c}7.11 \mathrm{~s} ; \\
{ }^{3} J_{\mathrm{PtH}} 35\end{array}$ & $\begin{array}{c}7.25 \mathrm{~s} ; \\
{ }^{3} J_{\mathrm{PtH}} 35\end{array}$ \\
\hline $\mathrm{H} 7 \mathrm{a}$ & $\begin{array}{c}3.33 \mathrm{dd} ; \\
{ }^{2} J 15 ;{ }^{3} J 7\end{array}$ & $\begin{array}{c}3.15 \mathrm{~m} ; \\
\text { ov. }\end{array}$ & 2.68 ov. & $\begin{array}{c}2.87 \mathrm{~d} ;{ }^{2} J 17 \\
{ }^{3} J_{\mathrm{PtH}} 95\end{array}$ & $\begin{array}{l}2.86 \mathrm{~d} ;{ }^{2} J 17 ; \\
{ }^{3} J_{\mathrm{PtH}} 95\end{array}$ & $\begin{array}{c}2.80 \mathrm{~d} ;{ }^{2} J 17 ; \\
{ }^{3} J_{\mathrm{PtH}} 95\end{array}$ \\
\hline $\mathrm{H} 7 \mathrm{~b}$ & $\begin{array}{c}3.63 \mathrm{dd} ; \\
{ }^{2} J 15 ;{ }^{3} J 7\end{array}$ & $\begin{array}{c}3.62 \mathrm{~m} ; \\
\text { ov. }\end{array}$ & $\begin{array}{l}3.59 \mathrm{dd} ; \\
{ }^{2} J 7 ;{ }^{3} \mathrm{~J} 6\end{array}$ & $\begin{array}{c}3.79 \mathrm{dd} ; \\
{ }^{2} J 17 ;{ }^{3} J 6\end{array}$ & $\begin{array}{l}3.67 \mathrm{dd} ; \\
{ }^{2} J 7 ;{ }^{3} J 6\end{array}$ & $\begin{array}{c}3.73 \mathrm{dd} ; \\
{ }^{2} J 17 ;{ }^{3} J 6\end{array}$ \\
\hline H8 & $\begin{array}{l}5.78 \mathrm{~m} ; \\
{ }^{2} J_{\mathrm{PtH}} 70\end{array}$ & $\begin{array}{l}5.77 \mathrm{~m} ; \\
{ }^{2} J_{\mathrm{PtH}} 70\end{array}$ & $\begin{array}{l}5.02 \mathrm{~m} ; \\
{ }^{2} J_{\mathrm{PtH}} 75\end{array}$ & $\begin{array}{l}5.03 \mathrm{~m} ; \\
{ }^{2} J_{\mathrm{PtH}} 75\end{array}$ & $\begin{array}{l}5.01 \mathrm{~m} ; \\
{ }^{2} J_{\mathrm{PtH}} 75\end{array}$ & $\begin{array}{l}5.00 \mathrm{~m} ; \\
{ }^{2} J_{\mathrm{PtH}} 75\end{array}$ \\
\hline H9cis & $\begin{array}{c}4.92 \mathrm{~d} ;{ }^{3} J 8 \\
{ }^{2} J_{\mathrm{PtH}} 70\end{array}$ & $\begin{array}{c}4.89 \mathrm{~d} ;{ }^{3} J 8 \\
{ }^{2} J_{\mathrm{PtH}} 70\end{array}$ & $\begin{array}{c}4.44 \mathrm{~d} ;{ }^{3} J 7.5 ; \\
{ }^{2} J_{\mathrm{PtH}} 75\end{array}$ & $\begin{array}{c}4.27 \mathrm{~d} ;{ }^{3} J 6.5 \\
{ }^{2} J_{\mathrm{PtH}} 75\end{array}$ & $\begin{array}{c}4.36 \mathrm{~d} ;{ }^{3} J 7.5 \\
{ }^{2} J_{\mathrm{PtH}} 75\end{array}$ & $\begin{array}{l}4.40 \mathrm{~d} \\
3_{J} 7.5\end{array}$ \\
\hline H9trans & $\begin{array}{l}4.89 \mathrm{~d} ;{ }^{3} J 14 \\
{ }^{2} J_{\mathrm{PtH}} 70\end{array}$ & $\begin{array}{l}4.87 \mathrm{~d} ;{ }^{3} J 14 \\
{ }^{2} J_{\mathrm{PtH}} 70\end{array}$ & $\begin{array}{l}3.53 \mathrm{~d} ;{ }^{3} J 13 \\
{ }^{2} J_{\mathrm{PtH}} 75\end{array}$ & $\begin{array}{l}3.83 \mathrm{~d} \\
{ }^{3} J 13.5\end{array}$ & $\begin{array}{c}3.83 \mathrm{~d} ;{ }^{3} J 13.5 ; \\
{ }^{2} J_{\mathrm{PtH}} 75\end{array}$ & $\begin{array}{l}3.85 \mathrm{~d} ; \\
{ }^{3} J 14\end{array}$ \\
\hline H12 & $9.14 \mathrm{~d} ;{ }^{3} J 5$ & - & - & 8 & $8.62 \mathrm{~d} ;{ }^{3} J 5$ & - \\
\hline $\mathrm{H} 12 \mathrm{a}$ & - & $3.17 \mathrm{~s}$ & $2.68 \mathrm{~s}$ & $9.81 \mathrm{~s}$ & - & $2.69 \mathrm{~s}$ \\
\hline H13 & $\begin{array}{c}7.68 \mathrm{dd} ; \\
{ }^{3} J 8.5 ;{ }^{3} J 5\end{array}$ & $\begin{array}{l}7.42 \mathrm{~d} ; \\
{ }^{3} J 8.5\end{array}$ & $\begin{array}{l}7.42 \mathrm{~d} ; \\
{ }^{3} J 8.5\end{array}$ & $\begin{array}{l}8.01 \mathrm{~d} ; \\
{ }^{3} J 8.5\end{array}$ & $\begin{array}{c}7.84 \mathrm{dd} ; \\
{ }^{3} J 8.5 ;{ }^{3}{ }^{2} 5\end{array}$ & $\begin{array}{l}7.35 \mathrm{~d} ; \\
{ }^{3} J 8.5\end{array}$ \\
\hline H14 & $\begin{array}{l}8.75 \mathrm{~d} ; \\
{ }^{3} J 8.5\end{array}$ & $\begin{array}{l}8.56 \mathrm{~d} \\
{ }^{3} J 8.5\end{array}$ & $\begin{array}{l}8.29 \mathrm{~d} \\
{ }^{3} J 8.5\end{array}$ & $\begin{array}{l}8.43 \mathrm{~d} ; \\
{ }^{3} J 8.5\end{array}$ & $\begin{array}{l}8.75 \mathrm{~d} ; \\
{ }^{3} J 8.5\end{array}$ & $\begin{array}{l}8.49 \mathrm{~d} \\
{ }^{3} J 8.5\end{array}$ \\
\hline H15 & - & - & $\begin{array}{l}7.01 \mathrm{dd} \\
{ }^{3} J 8 ;{ }^{4} J 1\end{array}$ & $\begin{array}{l}7.12 \mathrm{~d} ; \\
{ }^{3} \mathrm{~J} 8\end{array}$ & - & - \\
\hline H16 & $7.64 \mathrm{~s}$ & $7.54 \mathrm{~s}$ & $\begin{array}{l}7.38 \mathrm{t} ; \\
{ }^{3} \mathrm{~J} 8\end{array}$ & $\begin{array}{c}7.69 t ; \\
{ }^{3} J 8\end{array}$ & & $7.66 \mathrm{~s}$ \\
\hline H17 & - & - & $\begin{array}{c}6.98 \mathrm{dd} \\
{ }^{3} J 7.5 ; ;^{4} J 1\end{array}$ & $\begin{array}{c}6.29 \mathrm{dd} ; \\
{ }^{3} J 7.5 ;{ }^{4} J 1\end{array}$ & - & - \\
\hline
\end{tabular}


Table 4. The cell in vitro cytotoxicity of examined compounds, $\mathrm{IC}_{50}(\mu \mathrm{M})$.

\begin{tabular}{|l|c|c|c|c|}
\hline \multicolumn{1}{|c|}{ Cell line } & KB & Hep-G2 & Lu & MCF-7 \\
\hline$\left[\mathrm{Pt}(\mathrm{Saf}-1 \mathrm{H})\left(5,7-\mathrm{Cl}_{2}\right.\right.$-8-O-quinoline $\left.)\right](\mathbf{7})$ & 4.2 & 2.7 & 4.3 & 3.6 \\
\hline$[\mathrm{Pt}($ Eteug-1H)(2-Me-8-O-quinoline) $](\mathbf{9})$ & $>212$ & $>212$ & - & - \\
\hline$[\mathrm{Pt}($ Eteug-1H)(5,7-Cl 2 -8-O-quinoline) $](\mathbf{1 1})$ & 121.3 & 25.3 & - & - \\
\hline$[\mathrm{Pt}($ Eteug-1H)(5,7-Cl 2 -2-Me-8-O-quinoline) $](\mathbf{1 2})$ & 16.4 & 74.8 & 66.5 & 22.0 \\
\hline 8-HO-quinoline & 37.9 & 43.5 & 62.9 & 40.4 \\
\hline
\end{tabular}


<smiles></smiles><smiles>[X]c1ccc(OCC(=O)OC)c(OC)c1</smiles><smiles>C=P1(Cl)CCc2cc3c(cc21)OCO3</smiles>

$\mathrm{K}\left[\mathrm{PtCl}_{3}(\mathrm{Saf})\right]$ $\mathrm{K}\left[\mathrm{PtCl}_{3}(\right.$ Meteug $\left.)\right]$

$\left[\mathrm{Pt}_{2} \mathrm{Cl}_{2}(\mathrm{Saf}-1 \mathrm{H})_{2}\right]$

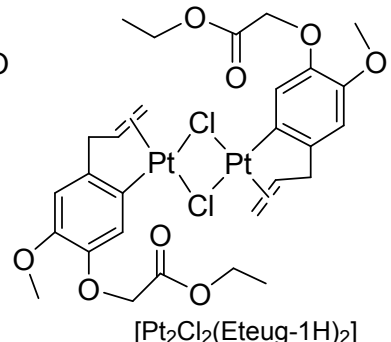

Figure 1. Starting organoplatinum(II) complexes.<smiles>CC(C)c1cc2c(cc1Cl)OCO2</smiles>

(1)<smiles>CC(CCl)Cc1cc2c(cc1CCCl)OCO2</smiles>

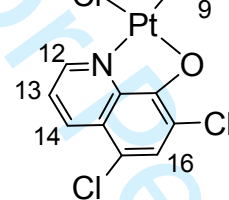

(2)

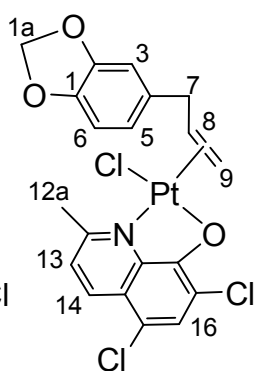

(3)

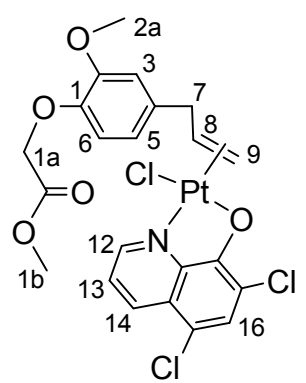

(4)

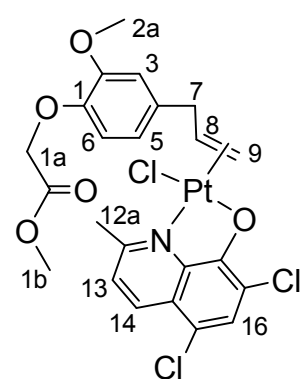

(5)

Figure 2. Structure of complexes 1-5 (the numeration on these structures is specifically used for NMR analysis).

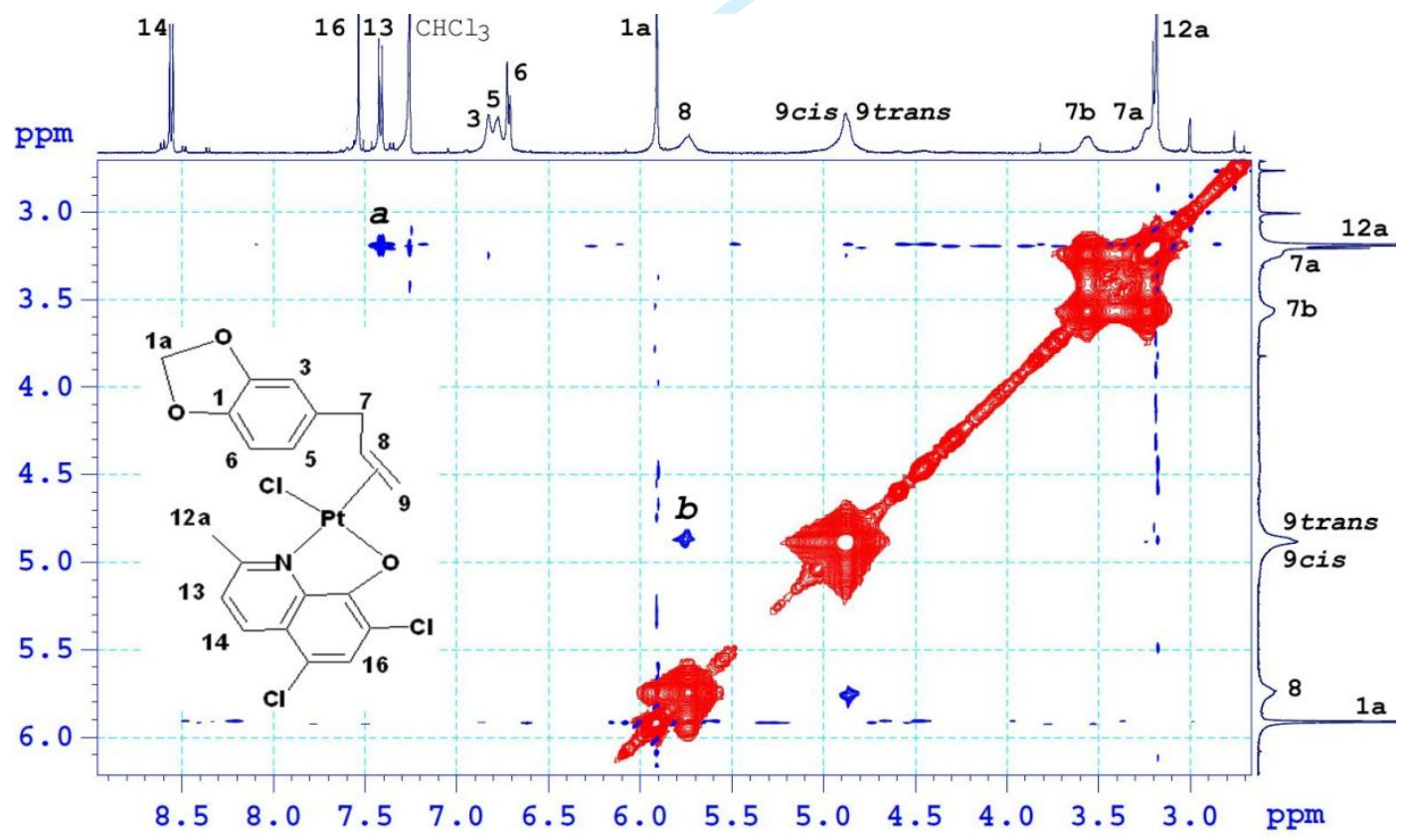

Figure 3. NOESY spectrum of complex 3. 


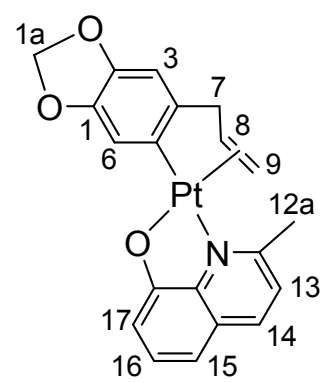

(6)

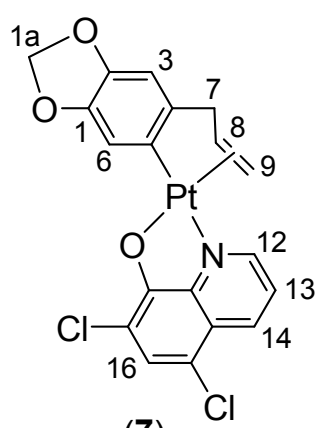

(7)

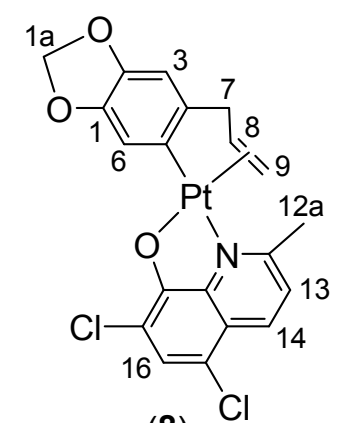

(8)

Figure 4. Structure of complexes 6-8.

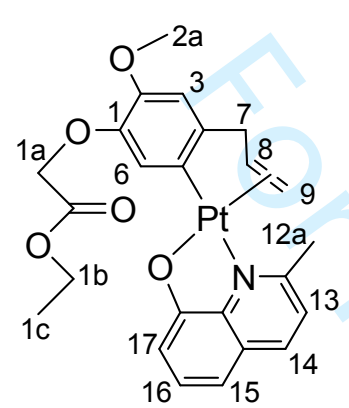

(9)

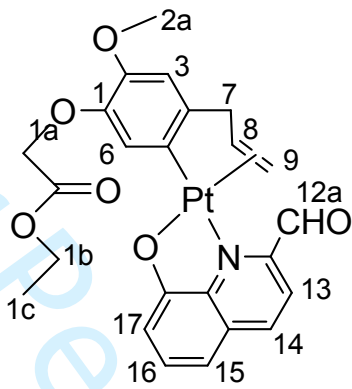

(10)

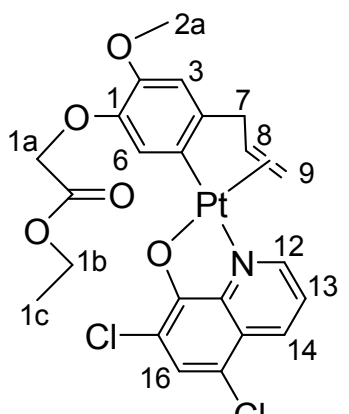

(11)

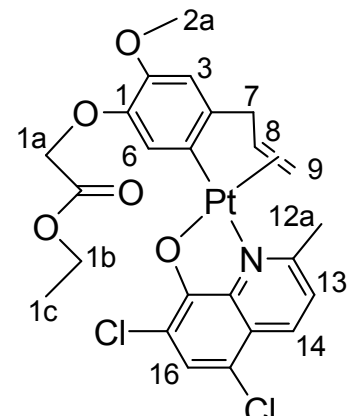

(12)

Figure 5. Structure of complexes 9-12 (the numeration on these structures is specifically used for NMR analysis).

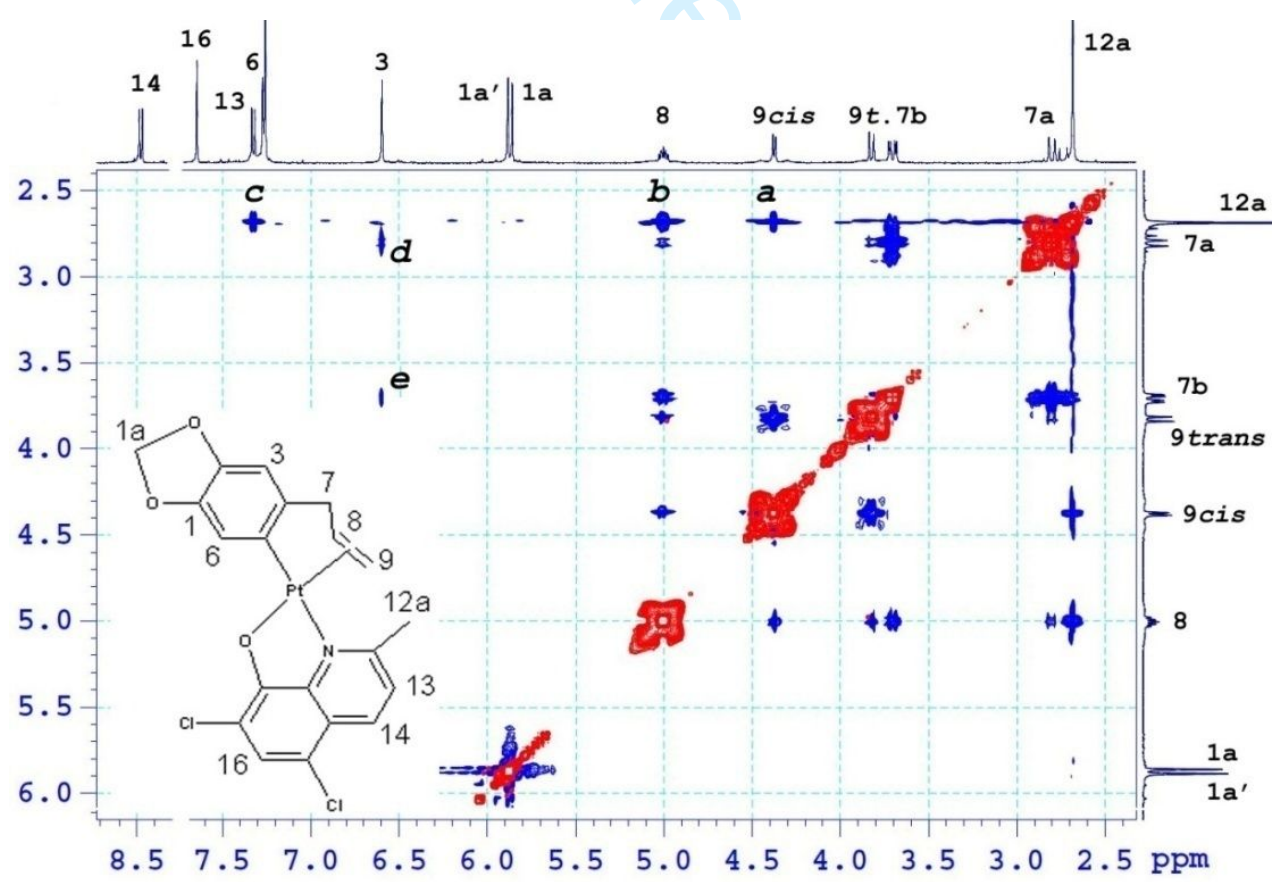

Figure 6. NOESY spectrum of complex 8 . 


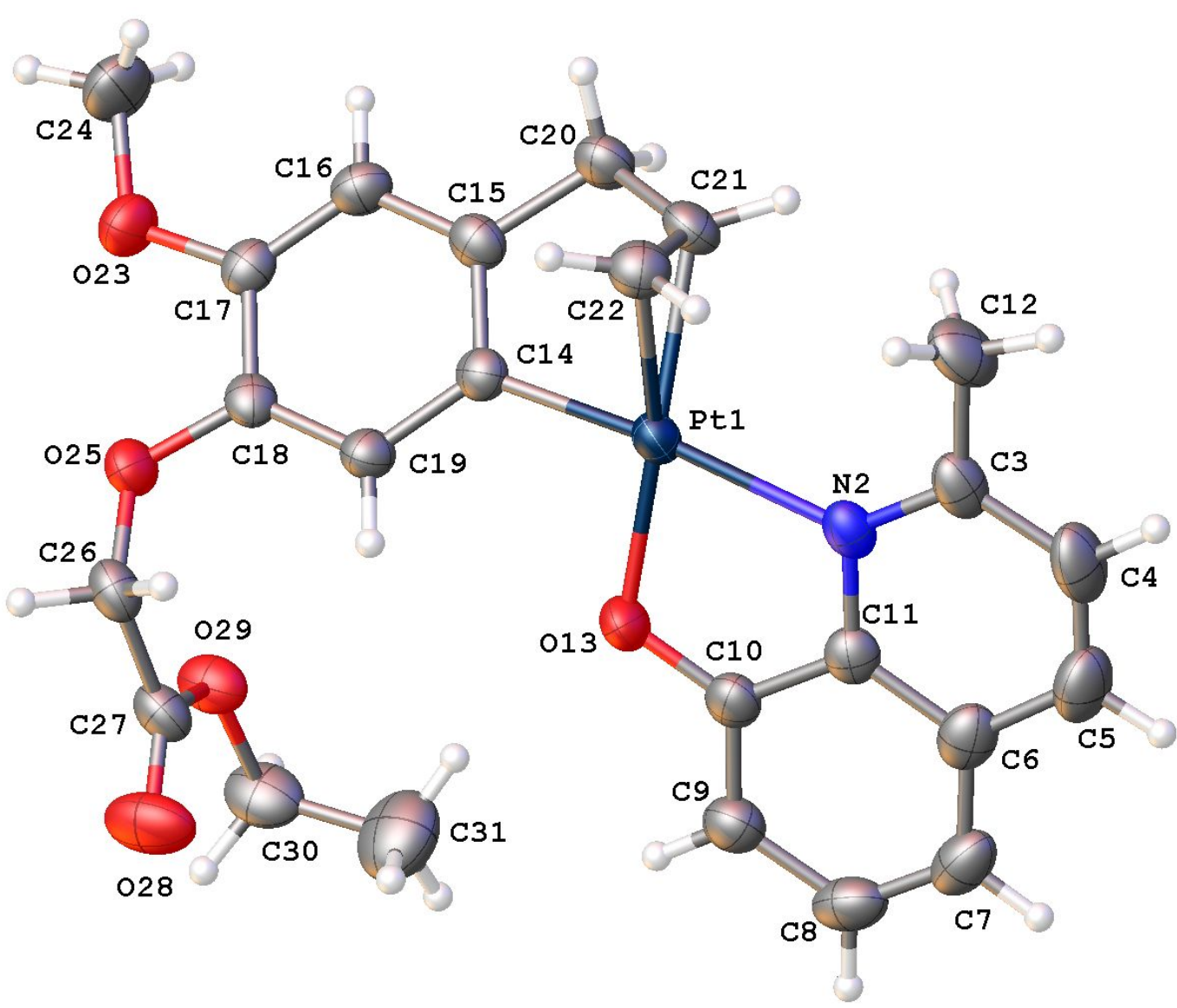

Figure 7. Molecular structure of $\mathbf{9}$, with atom labelling scheme and displacement ellipsoids drawn at the $50 \%$ probability level. 


\section{checkCIF/PLATON report}

Structure factors have been supplied for datablock(s) hai39

THIS REPORT IS FOR GUIDANCE ONLY. IF USED AS PART OF A REVIEW PROCEDURE FOR PUBLICATION, IT SHOULD NOT REPLACE THE EXPERTISE OF AN EXPERIENCED CRYSTALLOGRAPHIC REFEREE.

No syntax errors found. CIF dictionary Interpreting this report

\section{Datablock: hai39}

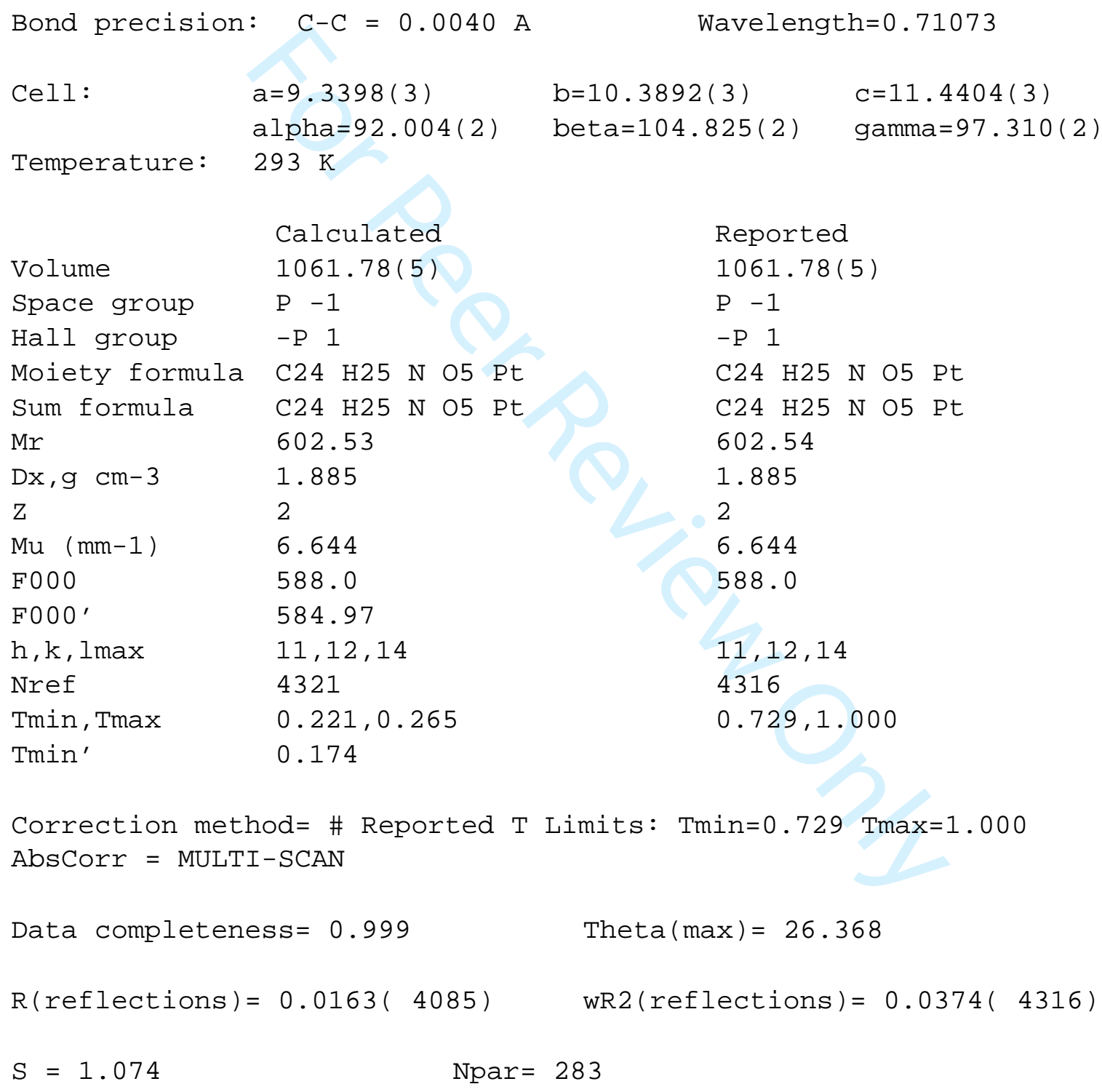


Alert level C

CRYSC01_ALERT_1_C The word below has not been recognised as a standard identifier. yellowish

\section{Alert level G}

PLAT012_ALERT_1_G No__shelx_res_checksum Found in CIF .....

PLAT154_ALERT_1_G The s.u.'s on the Cell Angles are Equal ... (Note)

PLAT199_ALERT_1_G Reported_cell_measurement_temperature ..... (K)

PLAT200_ALERT_1_G Reported_diffrn_ambient_temperature ..... (K)

PLAT794_ALERT_5_G Tentative Bond Valency for Pt1 (II) .

PLAT910_ALERT_3_G Missing \# of FCF Reflection(s) Below Theta(Min). PLAT933_ALERT_2_G Number of OMIT Records in Embedded.res File... PLAT978_ALERT_2_G Number C-C Bonds with Positive Residual Density.
Please Check
0.002 Degree
293 Check
293 Check
2.14 Info
4 Note
2 Note
5 Info

0 ALERT level $\mathbf{A}=$ Most likely a serious problem - resolve or explain

0 ALERT level $\mathbf{B}=\mathrm{A}$ potentially serious problem, consider carefully

1 ALERT level $\mathbf{C}=$ Check. Ensure it is not caused by an omission or oversight

8 ALERT level $\mathbf{G}=$ General information/check it is not something unexpected

5 ALERT type 1 CIF construction/syntax error, inconsistent or missing data

2 ALERT type 2 Indicator that the structure model may be wrong or deficient

1 ALERT type 3 Indicator that the structure quality may be low

0 ALERT type 4 Improvement, methodology, query or suggestion

1 ALERT type 5 Informative message, check

It is advisable to attempt to resolve as many as possible of the alerts in all categories. Often the minor alerts point to easily fixed oversights, errors and omissions in your CIF or refinement strategy, so attention to these fine details can be worthwhile. In order to resolve some of the more serious problems it may be necessary to carry out additional measurements or structure refinements. However, the purpose of your study may justify the reported deviations and the more serious of these should normally be commented upon in the discussion or experimental section of a paper or in the "special_details" fields of the CIF. checkCIF was carefully designed to identify outliers and unusual parameters, but every test has its limitations and alerts that are not important in a particular case may appear. Conversely, the absence of alerts does not guarantee there are no aspects of the results needing attention. It is up to the individual to critically assess their own results and, if necessary, seek expert advice.

\section{Publication of your CIF in IUCr journals}

A basic structural check has been run on your CIF. These basic checks will be run on all CIFs submitted for publication in IUCr journals (Acta Crystallographica, Journal of Applied Crystallography, Journal of Synchrotron Radiation); however, if you intend to submit to Acta Crystallographica Section C or E or IUCrData, you should make sure that full publication checks are run on the final version of your CIF prior to submission.

\section{Publication of your CIF in other journals}

Please refer to the Notes for Authors of the relevant journal for any special instructions relating to CIF submission. 


\section{PLATON version of 14/07/2018; check.def file version of $05 / 06 / 2018$}

Datablock hai39 - ellipsoid plot

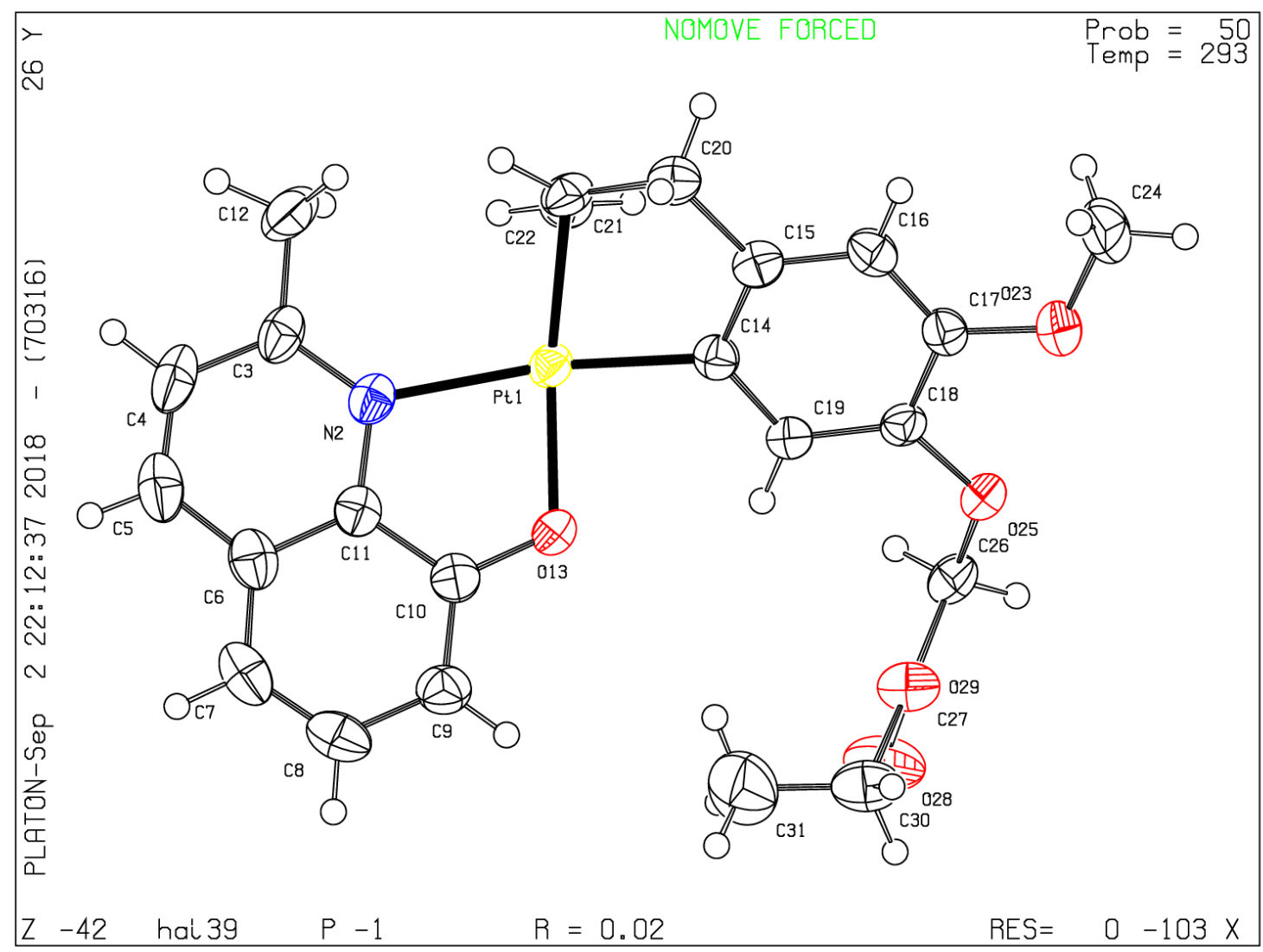

